\title{
Singular Non-circular Complex Elliptically Symmetric Distributions: New Results and Applications
}

\author{
Habti Abeida \\ Department of Electrical Engineering, College of Engineering, Taif University, Al-Haweiah, 21974, Saudi Arabia
}

\begin{abstract}
Received September 6, 2021; Revised October 25, 2021; Accepted November 11, 2021
Cite This Paper in the following Citation Styles

(a): [1] HHabti Abeida, "Singular Non-circular Complex Elliptically Symmetric Distributions: New Results and Applications," Mathematics and Statistics, Vol.9, No.6, pp. 1019-1033, 2021. DOI: 10.13189/ms.2021.090618

(b): Habti Abeida, (2021). Singular Non-circular Complex Elliptically Symmetric Distributions: New Results and Applications. Mathematics and Statistics, 9(6), 1019-1033. DOI: 10.13189/ms.2021.090618
\end{abstract}

Copyright $@ 2021$ by authors, all rights reserved. Authors agree that this article remains permanently open access under the terms of the Creative Commons Attribution License 4.0 International License

\begin{abstract}
Absolutely Continuous non-singular complex elliptically symmetric distributions (referred to as the nonsingular CES distributions) have been extensively studied in various applications under the assumption of nonsingularity of the scatter matrix for which the probability density functions (p.d.f's) exist. These p.d.f's, however, can not be used to characterize the CES distributions with a singular scatter matrix (referred to as the singular CES distributions). This paper presents a generalization of the singular real elliptically symmetric (RES) distributions studied by Díaz-García et al to singular CES distributions. An explicit expression of the p.d.f of a multivariate non-circular complex random vector with singular CES distribution is derived. The stochastic representation of the singular non-circular CES (NC-CES) distributions and the quadratic forms in NC-CES random vector are proved. As special cases, explicit expressions for the p.d.f's of multivariate complex random vectors with singular non-circular complex normal (NC-CN) and singular non-circular complex Compound-Gaussian (NC-CCG) distributions are also derived. Some useful properties of singular NC-CES distributions and their conditional distributions are derived. Based on these results, the p.d.f's of non-circular complex $t$-distribution, $K$ distribution, and generalized Gaussian distribution under singularity are presented. These general results degenerate to those of singular circular CES (C-CES) distributions when the pseudo-scatter matrix is equal to the zero matrix. Finally, these results are applied to the problem of estimating the parameters of a complex-valued non-circular multivariate linear model in the presence either of singular NC-CES or C-CES distributed noise terms by proposing widely linear estimators.
\end{abstract}

Keywords Non-singular CES Distributions, Singular CES Distributions, Circular Complex Random Vector, Non-circular Complex Random Vector, Circular/Non-circular Quadratic
Forms, Circular/Non-circular Complex-valued Linear Model

\section{Introduction}

Non-singular CES distributions have recently been the focus of active research in engineering applications involving non-Gaussian data models [1-8]. Very comprehensive reviews of non-singular C-CES and NC-CES distributions are given in $[5,9,10]$ and [11], respectively. The CES distribution includes various distributions, such as the circular complex normal (C-CN) distribution [12,13], NC-CN distribution [14, 22], complex t-distribution [1,9], K-distribution [10,23, 24], complex generalized Gaussian (CGG) distribution $[5,15,25]$. These distributions are characterized by the associated p.d.f.'s which exist for non-singular scatter or covariance matrix. However, a problem that has not been tackled completely is related to the p.d.f. of singular C-CES, NC-CES, C-CN, and NC-CN distributions, which are not unusual in theoretical and practical engineering problems. However, it was proved by [26] that the singular RES distributions have p.d.f's on a subspace of smaller dimension and equal to the rank of the scatter matrix. This same reference also gives an explicit expression for the p.d.f of singular RES distributions.

Complex-valued signals are widely used for modeling many systems in a wide range of fields (e.g., optics, communications, radar, and biomedicine). Linear solutions for complex-valued signals have been studied in detail in the literature for both circular and non-circular complex signals. Among these solutions, the linear and widely linear minimum mean-squared error (LMMSE and WLMMSE) estimators introduced in [27,28] work under the assumption that the covariance matrix of mea- 
surement is non-singular. The performance of these estimators are compared in $[29,30]$ and widely used in many practical applications [31-34]. However, these estimators can not be applied when the scatter or covariance matrix of the measurement data is singular. This paper presents the derivation of explicit expressions for the p.d.f.'s of multivariate singular C-CES, NCCES, C-CN and NC-CN distributed random variables (r.v.'s) following the reasoning proposed in [26]. Stochastic representations of singular C-CES distributions, singular NC-CES distributions and quadratic forms in singular C-CES and NCCES r.v.'s are also given. Some useful properties of singular NC-CES distributions and their conditional distributions are derived. The complex t-distribution, K-distribution, and generalized Gaussian distribution associated with singular multivariate CES r.v.'s are also derived. These results are applied to the problem of estimating the parameters of a complex-valued linear model in the presence of either singular NC-CES or CCES distributed noise terms and followed by the derivation of widely linear estimators.

The remainder of this paper is organized as follows. Section 2 presents a brief overview of general characteristics of the continuous non-singular NC-CES distributions, followed by the derivation of the p.d.f. and the stochastic representation of singular NC-CES distributions. In the same section, some useful properties of singular NC-CES distributions and their conditional distributions are proved. Section 3 provides the p.d.f of singular NC-CN distribution as a special case of NC-CES distributions. Section 4 introduces practical singular circular complex compound Gaussian (C-CCG) and singular NC-CCG distributions, followed by the derivation of the stochastic representation of the quadratic forms in singular CCCG and NC-CCG distributions. Section 5 presents the complex $t$-distribution, $K$-distribution and the CGG distribution associated to multivariate singular C-CES and NC-CES r.v.'s. Section 6 derives the singular widely linear mean square estimation of a signal from singular distributed measurement data vector. The problem of parametric estimation of complexvalued linear models with singular C-CES and NC-CES distributed error terms are examined in section 7 where the associated residuals of the widely linear estimators are shown to have singular CES distributions. Finally, conclusion is given in section 8 .

The following notations are used throughout the paper. Matrices and vectors are represented by bold upper case and bold lower case characters, respectively. $\mathbf{I}$ is the identity matrix. Vectors are by default in column orientation, while $T, H, *$ and \# stand for transpose, conjugate transpose, conjugate and MoorePenrose inverse,respectively. $\mathrm{E}(),. \operatorname{Tr}(),. \operatorname{rank}(),. \Re($. and $\|$.$\| are the expectation, trace, rank, real part, and norm op-$ erators, respectively. $\operatorname{Cov}(\mathbf{z}) \stackrel{\text { def }}{=}(\mathbf{z}-\mathrm{E}(\mathbf{z}))(\mathbf{z}-\mathrm{E}(\mathbf{z}))^{H}$ and $\operatorname{pcov}(\mathbf{z}) \stackrel{\text { def }}{=}(\mathbf{z}-\mathrm{E}(\mathbf{z}))(\mathbf{z}-\mathrm{E}(\mathbf{z}))^{T}$ are respectively the covariance matrix and the pseudo-covariance matrix of a complex r.v. z. Symbol $=_{d}$ means equal in distribution and $U\left(\mathbb{C} S^{m}\right)$ denotes the uniform distribution on the unit complex-sphere $\mathbb{C} S^{m} \stackrel{\text { def }}{=}\left\{\mathbf{z} \in \mathbb{C}:\|z\|^{2}=1\right\}$. $(\operatorname{Ker} \boldsymbol{\Sigma})^{\perp}$ is the complement orthogonal of kernel space (or null space) of $\boldsymbol{\Sigma}$.

\section{Singular and non-singular non- circular complex elliptical distribu- tion}

This section firstly briefly reviews of non-singular NC-CES distributions (called also Generalized CES distributions) presented in [11], secondly the main characteristics of singular NC-CES distributions are proven, i.e., singular with respect to Lebesgue measure as the scatter matrix is rank-deficient, by presenting an explicit expression of the p.d.f. of singular NCCES distributions that exist on a subspace. Finally we provide some useful properties of singular NC-CES distributions and their conditional distributions.

A r.v. $\mathbf{z} \in \mathbb{C}^{m}$ is said to have NC-CES distribution if its characteristic function (c.f.) is

$$
\Phi(\mathbf{z})=\exp \left\{j \Re\left(\mathbf{z}^{H} \boldsymbol{\mu}\right)\right\} \phi\left(\frac{1}{2}\left\{\mathbf{z}^{H} \mathbf{\Sigma} \mathbf{z}+\Re\left[\mathbf{z}^{H} \boldsymbol{\Omega} \mathbf{z}^{*}\right]\right\}\right),
$$

for some function $\phi: \mathbb{R}^{+} \rightarrow \mathbb{R}$, as the characteristic generator. Positive semi-definite Hermitian matrix $\boldsymbol{\Sigma} \in \mathbb{C}^{m \times m}$ is the scatter matrix, complex symmetric matrix $\Omega \in \mathbb{C}^{m \times m}$ denotes the pseudo-scatter matrix, with symmetry center $\boldsymbol{\mu} \in \mathbb{C}^{m}$. Note that the c.f. in (1) exists even though $\boldsymbol{\Sigma}$ is singular $(\operatorname{rank}(\boldsymbol{\Sigma})<m)$. In addition, $\boldsymbol{\Sigma}, \boldsymbol{\Omega}$ and $\phi($.$) do not uniquely$ define a particular NC-CES distribution, an additional scale constraint, either on $\boldsymbol{\Sigma}$ and $\boldsymbol{\Omega}$ either on $\phi($.$) , needs to be im-$ posed for identifiability purposes.

Therefore, an r.v. $\mathbf{z} \in \mathbb{C}^{m}$ has singular or non-singular NCCES distribution, depending on whether $\operatorname{rank}(\boldsymbol{\Sigma})=r<m$ or $r=m$, respectively. A singular NC-CES distributed r.v. will be denoted as $\mathbf{z} \sim \mathrm{EC}_{m}^{r}(\boldsymbol{\mu}, \boldsymbol{\Sigma}, \boldsymbol{\Omega}, \phi)$ and for non-singular CES distributed r.v. the superscript will be omitted in $\mathrm{EC}_{m}^{r}$. For clarity, the singular and non-singular NC-CES distributions are presented separately in the following subsections.

\subsection{Non-singular NC-CES distributions}

Suppose $\operatorname{rank}(\boldsymbol{\Sigma})=m$, which is a necessary condition for $\mathbf{z} \sim \mathrm{EC}_{m}(\boldsymbol{\mu}, \boldsymbol{\Sigma}, \boldsymbol{\Omega}, \phi)$ to be absolutely continues with respect to Lebesgue measure in $\mathbb{R}^{2 m}$, therefore the p.d.f. of $\mathbf{z}$ exist and can be expressed as [11],

$$
p(\mathbf{z})=c_{m, g}(\operatorname{det}(\tilde{\boldsymbol{\Gamma}}))^{-1 / 2} g(q(\mathbf{z})),
$$

where $c_{m, g}$ is a normalizing constant ensuring that $p(\mathbf{z})$ integrates to one and it is given by $c_{m, g} \stackrel{\text { def }}{=} 2\left(s_{m} \delta_{m, g}\right)^{-1}$, where $s_{m} \stackrel{\text { def }}{=} \frac{2 \pi^{m}}{\Gamma(m)}$ is the surface area of $\mathbb{C} S^{m}$, and $g($. is a non-negative function (density generator), which satisfies $\delta_{m, g}=\int_{0}^{\infty} u^{m-1} g(u) d u<\infty . q(\mathbf{z})$ has the quadratic form $q(\mathbf{z}) \stackrel{\text { def }}{=} \frac{1}{2}(\tilde{\mathbf{z}}-\tilde{\boldsymbol{\mu}})^{H} \tilde{\boldsymbol{\Gamma}}^{-1}(\tilde{\mathbf{z}}-\tilde{\boldsymbol{\mu}})$, where $\tilde{\mathbf{z}} \stackrel{\text { def }}{=}\left(\mathbf{z}^{H} \mathbf{z}^{T}\right)^{H}$, $\tilde{\boldsymbol{\mu}} \stackrel{\text { def }}{=}\left(\begin{array}{ll}\boldsymbol{\mu}^{H} & \boldsymbol{\mu}^{T}\end{array}\right)^{H}$ and $\tilde{\boldsymbol{\Gamma}} \stackrel{\text { def }}{=}\left(\begin{array}{cc}\boldsymbol{\Sigma} & \boldsymbol{\Omega} \\ \boldsymbol{\Omega}^{*} & \boldsymbol{\Sigma}^{*}\end{array}\right)$. In the absolutely continuous case, we use the notation $\mathbf{z} \sim \operatorname{EC}_{m}(\boldsymbol{\mu}, \boldsymbol{\Sigma}, \boldsymbol{\Omega}, g)$ in place of $\mathbf{z} \sim \operatorname{EC}_{m}(\boldsymbol{\mu}, \boldsymbol{\Sigma}, \boldsymbol{\Omega}, \phi)$. Note that the p.d.f. (2) depends on $\mathbf{z}$ only through the quadratic form $q(\mathbf{z})$. 
Since $\boldsymbol{\Sigma}$ and $\boldsymbol{\Omega}$ are hermitian positive definite and complex symmetric matrices, respectively, it follows from [35, Corollary 4.6.12(b)], that there exist a non-singular matrix $\mathbf{A} \in \mathbb{C}^{m \times m}$ such that $\boldsymbol{\Sigma}=\mathbf{A} \mathbf{A}^{H}$ and $\boldsymbol{\Omega}=\mathbf{A} \boldsymbol{\Delta} \mathbf{A}^{T}$ where $\boldsymbol{\Delta}=\operatorname{Diag}\left(\kappa_{1}, \ldots, \kappa_{m}\right)$ is a real diagonal matrix with nonnegative diagonal entries $\kappa_{k}$ for $k=1 \ldots m$. Let $\mathbf{v} \in \mathbb{C}^{m}$ be a r.v. obtained via an $\mathbb{R}$-linear transformation of $\mathbf{u} \sim U\left(\mathbb{C} S^{m}\right)$ as follows [5]:

$$
\mathbf{v}=\boldsymbol{\Delta}_{1} \mathbf{u}+\boldsymbol{\Delta}_{2} \mathbf{u}^{*}
$$

$\boldsymbol{\Delta}_{1} \stackrel{\text { def }}{=}\left(\frac{\boldsymbol{\Delta}_{+}+\boldsymbol{\Delta}_{-}}{2}\right), \boldsymbol{\Delta}_{2} \stackrel{\text { def }}{=}\left(\frac{\boldsymbol{\Delta}_{+}-\boldsymbol{\Delta}_{-}}{2}\right)$, where $\boldsymbol{\Delta}_{+}=$ $\sqrt{\mathbf{I}+\boldsymbol{\Delta}}$ and $\boldsymbol{\Delta}_{-}=\sqrt{\mathbf{I}-\boldsymbol{\Delta}} . \boldsymbol{\Delta}_{1}$ and $\boldsymbol{\Delta}_{2}$ satisfy $\boldsymbol{\Delta}_{1} \boldsymbol{\Delta}_{1}+$ $\Delta_{2} \Delta_{2}=$ I and $\Delta_{1} \Delta_{2}+\Delta_{2} \Delta=\Delta$. It follows from (3) that $\mathrm{E}\left(\mathbf{v} \mathbf{v}^{H}\right)=\mathrm{E}\left(\mathbf{u} \mathbf{u}^{H}\right)=\mathbf{I}$ and $\mathrm{E}\left(\mathbf{v} \mathbf{v}^{T}\right)=\boldsymbol{\Delta}$. In the sequel, this vector will be written as $\mathbf{v} \sim U_{\boldsymbol{\kappa}}\left(\mathbb{C} S^{m}\right)$ where $\boldsymbol{\kappa} \stackrel{\text { def }}{=}\left(\kappa_{1}, \ldots, \kappa_{m}\right)^{T}$. The stochastic representation provides the tool to generate r.v. deviating from the $\mathrm{EC}_{m}(\boldsymbol{\mu}, \boldsymbol{\Sigma}, \boldsymbol{\Omega}, g)$ distributions.

Result $1 \mathbf{z} \sim \mathrm{EC}_{m}(\boldsymbol{\mu}, \boldsymbol{\Sigma}, \boldsymbol{\Omega}, g)$ with $\operatorname{rank}(\boldsymbol{\Sigma})=m$ if and only if it admits the stochastic representation

$$
\mathbf{z}={ }_{d} \boldsymbol{\mu}+\mathcal{R} \mathbf{A} \mathbf{v}=\boldsymbol{\mu}+\mathcal{R}\left(\mathbf{A}_{1} \mathbf{u}+\mathbf{A}_{2} \mathbf{u}^{*}\right),
$$

where the non-negative real random variable $\mathcal{R} \stackrel{\text { def }}{=} \sqrt{\mathcal{Q}}$, called the modular variate, is independent of the complex r.v. $\mathbf{u}, \boldsymbol{\Sigma}=\mathbf{A}_{1} \mathbf{A}_{1}^{H}+\mathbf{A}_{2} \mathbf{A}_{2}^{H}$ and $\boldsymbol{\Omega}=\mathbf{A}_{1} \mathbf{A}_{2}^{T}+\mathbf{A}_{2} \mathbf{A}_{1}^{T}$ where $\mathbf{A}_{1} \stackrel{\text { def }}{=} \mathbf{A} \boldsymbol{\Delta}_{1}$ and $\mathbf{A}_{2} \stackrel{\text { def }}{=} \mathbf{A} \boldsymbol{\Delta}_{2}$.

The uniform spherical distribution can be obtained from a $\mathrm{C}$ $\mathrm{CN}$ distributed random vector, $\mathbf{y} \sim \mathbb{C N}_{m}(\mathbf{0}, \mathbf{I})$, when dividing it by its length, $\mathbf{u}={ }_{d} \frac{\mathbf{y}}{\|\mathbf{y}\|_{2}}$. Since $\delta_{m, g}<\infty$, the covariance matrix $\mathbf{R} \stackrel{\text { def }}{=} \operatorname{Cov}(\mathbf{z})$ and the pseudo covariance matrix $\mathbf{R}^{\prime} \stackrel{\text { def }}{=} \operatorname{pcov}(\mathbf{z})$ exist and respectively equal to the scatter matrix and pseudo-scatter matrix up to a positive real constant $c_{0}$ [11, Theorem 3], i.e., $\mathbf{R}=c_{0} \boldsymbol{\Sigma}$ and $\mathbf{R}^{\prime}=c_{0} \boldsymbol{\Omega}$. Nevertheless, the constant $c_{0}$ can be chosen to be equal to 1 , that is, if $\mathrm{E}\left(\mathcal{R}^{2}\right)=2 \operatorname{rank}(\boldsymbol{\Sigma})$. Note that while $\boldsymbol{\Sigma}$ always exists, $\mathbf{R}$ does not exist for some CES distributions (e.g. Cauchy distribution).

For the special case when $\Omega=\mathbf{O}$ (or equivalently $\Delta=\mathbf{O}$ ), the non-singular NC-CES distributions degenerate to the nonsingular C-CES distributions [5, 10], for which the c.f. (1) and p.d.f. (2) take the forms similar to the real case:

$$
\begin{aligned}
\Phi(\mathbf{z}) & =\exp \left\{j \Re\left(\mathbf{z}^{H} \boldsymbol{\mu}\right)\right\} \phi\left(\frac{1}{2} \mathbf{z}^{H} \boldsymbol{\Sigma} \mathbf{z}\right), \\
p(\mathbf{z}) & =c_{m, g} \operatorname{det}\left(\boldsymbol{\Sigma}^{-1}\right) g(q(\mathbf{z})),
\end{aligned}
$$

where $q(\mathbf{z}) \stackrel{\text { def }}{=}(\mathbf{z}-\boldsymbol{\mu})^{H} \boldsymbol{\Sigma}^{-1}(\mathbf{z}-\boldsymbol{\mu})$. It follows also from result 1 that the stochastic representation of non-singular $C$ CES distributed r.v. $\mathbf{z} \sim \mathrm{EC}_{m}(\boldsymbol{\mu}, \boldsymbol{\Sigma}, g)$ has the form

$$
\mathbf{z}={ }_{d} \boldsymbol{\mu}+\mathcal{R} \mathbf{A u},
$$

where $\mathbf{A} \in \mathbb{C}^{m \times m}$ is a non-singular matrix such that $\boldsymbol{\Sigma}=$ $\mathbf{A} \mathbf{A}^{H}$

\subsection{Singular NC-CES distributions}

The singular NC-CES distributions has still not been studied in the literature, in despite of the various studies that have been published. The results of this section are generalizations of [26] for the singular RES distribution to the singular NC-CES distributions, as the scatter matrix is singular with $\operatorname{rank}(\boldsymbol{\Sigma})=$ $r<m$. This section first proves an explicit expression of the p.d.f. for singular NC-CES distributions, and provides some useful properties of singular NC-CES distributions and their conditional distributions.

Note that the conditions of the scatter matrix $\boldsymbol{\Sigma}$ and the schur complement matrix $\boldsymbol{\Sigma}^{*}-\boldsymbol{\Omega}^{H} \boldsymbol{\Sigma}^{-1} \boldsymbol{\Omega}$ begin hermitian positive definite are necessary to ensure that the matrix $\tilde{\boldsymbol{\Gamma}}$ is hermitian positive definite [14]. However, if the matrix $\boldsymbol{\Sigma}$ is singular with $\operatorname{rank}(\boldsymbol{\Sigma})=r<m, \tilde{\boldsymbol{\Gamma}}$ is singular as well and the p.d.f in (2) has no meaning. Therefore the following question arises: Does the p.d.f. exist for singular NC-CES distributed r.v. $\mathbf{z} \sim$ $\mathrm{EC}_{m}^{r}(\boldsymbol{\mu}, \boldsymbol{\Sigma}, \boldsymbol{\Omega}, g)$ ? The answer is given by the result 2 where the p.d.f. exists on a subspace. To derive the p.d.f. of singular NC-CSE distributions we need the following lemma (proved in the Appendix A) that provides a factorization of the pseudoscatter matrix $\Omega$.

Lemma 1 Let $\operatorname{rank}(\boldsymbol{\Sigma})=r<m$ and $\operatorname{rank}(\Omega)=p \leq m$, the pseudo-scatter matrix $\Omega$ can be factorized as:

$$
\boldsymbol{\Omega}=\mathbf{U}_{r} \boldsymbol{\Lambda}_{r}^{\frac{1}{2}} \mathbf{V}_{p} \boldsymbol{\Delta}_{p} \mathbf{V}_{p}^{T} \boldsymbol{\Lambda}_{r}^{\frac{1}{2}} \mathbf{U}_{r}^{T}
$$

where $\boldsymbol{\Lambda}_{r}$ is a diagonal matrix containing the $r$ non-zero eigenvalues $\left\{\lambda_{k}\right\}_{k=1}^{r}$ of $\boldsymbol{\Sigma}$, and the columns of the complex matrix $\mathbf{U}_{r} \in \mathbb{C}^{m \times r}$ are the corresponding non-zero eigenvectors. $\mathbf{V}_{p} \in \mathbb{C}^{r \times p}$ is an $(r \times p)$ matrix with orthonormal columns and $\Delta_{p} \stackrel{\text { def }}{=} \operatorname{Diag}\left(\kappa_{1}, \ldots, \kappa_{p}\right)$ is $a(p \times p)$ complex-valued nonnegative diagonal matrix with $\kappa_{l} \neq 0$, and $\left|\kappa_{l}\right|<1$ for $l=1, \ldots, p$.

The following result (proved in the Appendix B) provides the p.d.f. of the singular NC-CES distributions.

Result 2 Let $\mathbf{z} \sim \mathrm{EC}_{m}^{r}(\boldsymbol{\mu}, \boldsymbol{\Sigma}, \boldsymbol{\Omega}, g)$ with parameters $\boldsymbol{\mu} \in \mathbb{C}^{m}$, $\boldsymbol{\Sigma} \in \mathbb{C}^{m \times m}$ is the scatter complex hermitian matrix assumed singular with $\operatorname{rank}(\boldsymbol{\Sigma})=r<m$ and $\boldsymbol{\Omega} \in \mathbb{C}^{m \times m}$ is the pseudo-scatter complex symmetric matrix with $\operatorname{rank}(\Omega)=p \leq$ $m$. In such case, the p.d.f. of a singular NC-CES distributed r.v. $\mathbf{z}$ is given by

$$
p(\mathbf{z})=c_{r, g} c_{\lambda, \kappa}^{r, p} g(q(\mathbf{z})),
$$

and

$$
\mathbf{z}-\boldsymbol{\mu} \in(\operatorname{Ker} \boldsymbol{\Sigma})^{\perp} \text { with probability } 1 \text { (w.p.1), }
$$

where $c_{\lambda, \kappa}^{r, p} \stackrel{\text { def }}{=}\left(\prod_{k=1}^{r} \lambda_{k}\right)^{-1}\left(\prod_{l=1}^{p}\left(1-\left|\kappa_{l}\right|^{2}\right)\right)^{-1 / 2}$ and $q(\mathbf{z})$ is a quadratic form $q(\mathbf{z}) \stackrel{\text { def }}{=} \frac{1}{2}(\tilde{\mathbf{z}}-\tilde{\boldsymbol{\mu}})^{H} \tilde{\boldsymbol{\Gamma}}^{\#}(\tilde{\mathbf{z}}-\tilde{\boldsymbol{\mu}})$.

Remark 1 It follows from lemma 1 that if $\operatorname{rank}(\boldsymbol{\Sigma})=$ $\operatorname{rank}(\boldsymbol{\Omega})=r$, there is a unitary matrix $\mathbf{V}_{r} \in \mathbb{C}^{r \times r}$ such that

$$
\boldsymbol{\Omega}=\mathbf{U}_{r} \boldsymbol{\Lambda}_{r}^{\frac{1}{2}} \mathbf{V}_{r} \boldsymbol{\Delta}_{r} \mathbf{V}_{r}^{T} \boldsymbol{\Lambda}_{r}^{\frac{1}{2}} \mathbf{U}_{r}^{T}
$$


where $\boldsymbol{\Delta}_{r}=\operatorname{Diag}(\boldsymbol{\kappa}), \boldsymbol{\kappa}=\left(\kappa_{1}, \ldots, \kappa_{r}\right)^{T} \in \mathbb{R}^{r}$ with $\kappa_{k} \neq 0$ for $k=1 \ldots r$. Let $\mathbf{W}_{r}$ be a $(r \times m)$ complex matrix defined as $\mathbf{W}_{r} \stackrel{\text { def }}{=} \mathbf{V}_{r}^{H} \boldsymbol{\Lambda}_{r}^{-\frac{1}{2}} \mathbf{U}_{r}^{H}$ which satisfies the following equalities:

$$
\mathbf{W}_{r} \boldsymbol{\Sigma} \mathbf{W}_{r}^{H}=\mathbf{I} \text { and } \mathbf{W}_{r} \boldsymbol{\Omega} \mathbf{W}_{r}^{T}=\boldsymbol{\Delta}_{r} .
$$

The following result extends result 1 to give a stochastic representation of a r.v. distributed as a singular NC-CES distribution.

Result 3 A r.v. z follows a singular NC-CES distribution, i.e., $\mathbf{z} \sim \mathrm{EC}_{m}^{r}(\boldsymbol{\mu}, \boldsymbol{\Sigma}, \boldsymbol{\Omega}, g)$ with $\operatorname{rank}(\boldsymbol{\Sigma})=\operatorname{rank}(\boldsymbol{\Omega})=r$ if and only if it admits the stochastic representation

$$
\mathbf{z}={ }_{d} \boldsymbol{\mu}+\mathcal{R} \mathbf{U}_{r} \boldsymbol{\Lambda}_{r}^{\frac{1}{2}} \mathbf{V}_{r} \mathbf{V}^{(r)}
$$

where the r.v. $\mathcal{R}$ is defined in (6) which is independent of $\mathbf{v}^{(r)} \sim$ $U_{\boldsymbol{\kappa}}\left(\mathbb{C} S^{r}\right)$ defined in (3), $\mathbf{U}_{r}$ and $\boldsymbol{\Lambda}_{r}$ are defined in (52) and $\mathbf{V}_{r}$ is defined in (10).

Note that the singular C-CES distribution is obtained if $\boldsymbol{\Omega}=$ $\mathbf{O}$, i.e., $\mathrm{EC}_{m}^{r}(\boldsymbol{\mu}, \boldsymbol{\Sigma}, g) \equiv \mathrm{EC}_{m}^{r}(\boldsymbol{\mu}, \boldsymbol{\Sigma}, \mathbf{O}, g)$. Therefore, result 2 can be simplified to the following result.

Result 4 Let $\mathbf{z} \sim \mathrm{EC}_{m}^{r}(\boldsymbol{\mu}, \boldsymbol{\Sigma}, g)$ with $\boldsymbol{\mu} \in \mathbb{C}^{m}, \boldsymbol{\Sigma} \in \mathbb{C}^{m \times m}$, $\boldsymbol{\Sigma} \geq \mathbf{O}$ and $\operatorname{rank}(\boldsymbol{\Sigma})=r<m$. In such case, the p.d.f. of $\mathbf{z}$ is given by

$$
p(\mathbf{z})=c_{r, g} c_{\lambda}^{r} g(\mathrm{q}(\mathbf{z}))
$$

and

$$
\mathbf{z}-\boldsymbol{\mu} \in(\operatorname{Ker} \boldsymbol{\Sigma})^{\perp} \text { w.p.l, }
$$

where $c_{\lambda}^{r} \stackrel{\text { def }}{=} \prod_{k=1}^{r} \lambda_{k}^{-1}$ and $\mathrm{q}(\mathbf{z})=(\mathbf{z}-\boldsymbol{\mu})^{H} \boldsymbol{\Sigma}^{\#}(\mathbf{z}-\boldsymbol{\mu})$.

The following corollary proved in Appendix C gives the distribution of the quadratic forms.

Corollary $\mathbf{1} \cdot$ Let $\mathbf{z} \sim \mathrm{EC}_{m}^{r}(\boldsymbol{\mu}, \boldsymbol{\Sigma}, \boldsymbol{\Omega}, g)$. Then

$$
(\tilde{\mathbf{z}}-\tilde{\boldsymbol{\mu}})^{H} \tilde{\boldsymbol{\Gamma}}^{\#}(\tilde{\mathbf{z}}-\tilde{\boldsymbol{\mu}})={ }_{d} 2 \mathcal{Q} .
$$

- Let $\mathbf{z} \sim \mathrm{EC}_{m}^{r}(\boldsymbol{\mu}, \boldsymbol{\Sigma}, g)$. Then

$$
(\mathbf{z}-\boldsymbol{\mu})^{H} \boldsymbol{\Sigma}^{\#}(\mathbf{z}-\boldsymbol{\mu})={ }_{d} \mathcal{Q} .
$$

The following result proved in Appendix D on the conditional distributions of singular NC-CES distributed r.v.'s will be used in the derivation of singular widely linear mean square estimation of a signal from singular distributed measurement data vector in section 6 .

Result 5 Let $\mathbf{z}=\left(\mathbf{z}_{1}^{H} \mathbf{z}_{2}^{H}\right)^{H} \sim \mathrm{EC}_{m}^{r}(\boldsymbol{\mu}, \boldsymbol{\Sigma}, \boldsymbol{\Omega}, g)$ and partition $\mu, \Sigma, \Omega$ into

$\boldsymbol{\mu}=\left(\begin{array}{l}\boldsymbol{\mu}_{1} \\ \boldsymbol{\mu}_{2}\end{array}\right), \boldsymbol{\Sigma}=\left(\begin{array}{ll}\boldsymbol{\Sigma}_{11} & \boldsymbol{\Sigma}_{12} \\ \boldsymbol{\Sigma}_{21} & \boldsymbol{\Sigma}_{22}\end{array}\right), \boldsymbol{\Omega}=\left(\begin{array}{ll}\boldsymbol{\Omega}_{11} & \boldsymbol{\Omega}_{12} \\ \boldsymbol{\Omega}_{21} & \boldsymbol{\Omega}_{22}\end{array}\right)$

where $\mathbf{z}_{1}$ and $\boldsymbol{\mu}_{1}$ are $(d \times 1)$ vectors $(d<m), \boldsymbol{\Sigma}_{11}$ and $\boldsymbol{\Omega}_{11}$ are $(d \times d)$ matrices, $\boldsymbol{\Sigma}_{22}$ and $\boldsymbol{\Omega}_{22}$ are $(n \times n)$ matrices (with $n=m-d)$. Also, assume that $\operatorname{rank}\left(\boldsymbol{\Sigma}_{11}\right)=r_{1} \leq d$ and $\operatorname{rank}\left(\boldsymbol{\Sigma}_{22}\right)=r_{2} \leq n$ with $r=r_{1}+r_{2}$. Then

$$
\begin{aligned}
& \cdot \mathbf{z}_{1} \sim \quad \operatorname{EC}_{d}^{r_{1}}\left(\boldsymbol{\mu}_{1}, \boldsymbol{\Sigma}_{11}, \boldsymbol{\Omega}_{11}, g\right) \quad \text { and } \quad \mathbf{z}_{2} \quad \sim \\
& \quad \mathrm{EC}_{n}^{r_{2}}\left(\boldsymbol{\mu}_{2}, \boldsymbol{\Sigma}_{22}, \boldsymbol{\Omega}_{22}, g\right) \\
& \text { - } \mathbf{z}_{1} \mid \mathbf{z}_{2} \sim \mathrm{EC}_{d}^{r_{1.2}}\left(\boldsymbol{\mu}_{z_{1} \mid z_{2}}, \boldsymbol{\Sigma}_{11.2}, \boldsymbol{\Omega}_{11.2}, g_{1 \mid 2}\right)
\end{aligned}
$$

with

$$
\begin{aligned}
\boldsymbol{\mu}_{z_{1} \mid z_{2}} & =\boldsymbol{\mu}_{1}+\mathbf{E}\left(\mathbf{z}_{2}-\boldsymbol{\mu}_{2}\right)+\mathbf{F}\left(\mathbf{z}_{2}^{*}-\boldsymbol{\mu}_{2}^{*}\right) \\
\boldsymbol{\Sigma}_{11.2} & =\boldsymbol{\Sigma}_{11}-\mathbf{E} \boldsymbol{\Sigma}_{12}^{H}-\mathbf{F} \boldsymbol{\Omega}_{12}^{H} \\
\boldsymbol{\Omega}_{11.2} & =\boldsymbol{\Omega}_{11}-\mathbf{E} \boldsymbol{\Omega}_{12}^{T}-\mathbf{F} \boldsymbol{\Sigma}_{12}^{T}
\end{aligned}
$$

where

$$
\begin{aligned}
& \mathbf{E} \stackrel{\text { def }}{=}\left(\boldsymbol{\Sigma}_{12}-\boldsymbol{\Omega}_{12} \boldsymbol{\Sigma}_{22}^{* \#} \boldsymbol{\Omega}_{22}^{H}\right) \mathbf{P}_{z_{2}}^{* \#} \\
& \mathbf{F} \stackrel{\text { def }}{=}\left(\boldsymbol{\Omega}_{12}-\boldsymbol{\Sigma}_{12} \boldsymbol{\Sigma}_{22}^{\#} \boldsymbol{\Omega}_{22}\right) \mathbf{P}_{z_{2}}^{\#}
\end{aligned}
$$

$\mathbf{P}_{z_{2}} \stackrel{\text { def }}{=} \boldsymbol{\Sigma}_{22}^{*}-\boldsymbol{\Omega}_{22}^{H} \boldsymbol{\Sigma}_{22}^{\#} \boldsymbol{\Omega}_{22}, r_{1.2}=\operatorname{rank}\left(\boldsymbol{\Sigma}_{11.2}\right), g_{1 \mid 2}(t)=$ $g\left(t+q_{2}\right), q_{2}=\frac{1}{2}\left(\tilde{\mathbf{z}}_{2}-\tilde{\boldsymbol{\mu}}_{2}\right)^{H} \tilde{\boldsymbol{\Gamma}}_{z_{2}}^{\#}\left(\tilde{\mathbf{z}}_{2}-\tilde{\boldsymbol{\mu}}_{2}\right), \tilde{\mathbf{z}}_{2}=\left(\mathbf{z}_{2}^{T} \mathbf{z}_{2}^{H}\right)^{T}$, $\tilde{\boldsymbol{\mu}}_{2}=\left(\begin{array}{ll}\boldsymbol{\mu}_{2}^{T} & \boldsymbol{\mu}_{2}^{H}\end{array}\right)^{T}$ and $\tilde{\boldsymbol{\Gamma}}_{z_{2}} \stackrel{\text { def }}{=}\left(\begin{array}{cc}\boldsymbol{\Sigma}_{22} & \boldsymbol{\Omega}_{22} \\ \boldsymbol{\Omega}_{22}^{*} & \boldsymbol{\Sigma}_{22}^{*}\end{array}\right)$.

Note that the singular C-CES distribution is obtained if $\Omega=\mathbf{O}$ and the result 5 degenerates to the following corollary.

Corollary 2 Let $\mathbf{z}=\left(\mathbf{z}_{1}^{H} \mathbf{z}_{2}^{H}\right)^{H} \sim \operatorname{EC}_{m}^{r}(\boldsymbol{\mu}, \boldsymbol{\Sigma}, g) \equiv$ $\mathrm{EC}_{m}^{r}(\boldsymbol{\mu}, \boldsymbol{\Sigma}, \mathbf{O}, g)$ and partition $\boldsymbol{\mu}$ and $\boldsymbol{\Sigma}$ into

$$
\boldsymbol{\mu}=\left(\begin{array}{l}
\boldsymbol{\mu}_{1} \\
\boldsymbol{\mu}_{2}
\end{array}\right) \text { and } \boldsymbol{\Sigma}=\left(\begin{array}{ll}
\boldsymbol{\Sigma}_{11} & \boldsymbol{\Sigma}_{12} \\
\boldsymbol{\Sigma}_{21} & \boldsymbol{\Sigma}_{22}
\end{array}\right)
$$

where $\mathbf{z}_{1}$ and $\boldsymbol{\mu}_{1}$ are $(d \times 1)$ vectors $(d<m), \boldsymbol{\Sigma}_{11}$ is a $(d \times d)$ matrix, $\boldsymbol{\Sigma}_{22}$ is $a(n \times n)$ matrix (with $n=m-d$ ). Also, assume that $\operatorname{rank}\left(\boldsymbol{\Sigma}_{11}\right)=r_{1} \leq d$ and $\operatorname{rank}\left(\boldsymbol{\Sigma}_{22}\right)=r_{2} \leq n$ with $r=r_{1}+r_{2}$. Then

$$
\begin{aligned}
& \cdot \mathbf{z}_{1} \sim \operatorname{EC}_{d}^{r_{1}}\left(\boldsymbol{\mu}_{1}, \boldsymbol{\Sigma}_{11}, g\right) \text { and } \mathbf{z}_{2} \sim \operatorname{EC}_{n}^{r_{2}}\left(\boldsymbol{\mu}_{2}, \boldsymbol{\Sigma}_{22}, g\right) \\
& \text { - } \mathbf{z}_{1} \mid \mathbf{z}_{2} \sim \mathrm{EC}_{d}^{r_{1.1}}\left(\boldsymbol{\mu}_{z_{1} \mid z_{2}}, \boldsymbol{\Sigma}_{1.1}, g_{1 \mid 2}\right)
\end{aligned}
$$

with

$$
\begin{aligned}
\boldsymbol{\mu}_{z_{1} \mid z_{2}} & =\boldsymbol{\mu}_{1}+\boldsymbol{\Sigma}_{12} \boldsymbol{\Sigma}_{22}^{\#}\left(\mathbf{z}_{2}-\boldsymbol{\mu}_{2}\right) \\
\boldsymbol{\Sigma}_{1.1} & =\boldsymbol{\Sigma}_{11}-\boldsymbol{\Sigma}_{12} \boldsymbol{\Sigma}_{22}^{\#} \boldsymbol{\Sigma}_{12}^{H}
\end{aligned}
$$

where $r_{1.1}=\operatorname{rank}\left(\boldsymbol{\Sigma}_{1.1}\right)$ and $g_{1 \mid 2}(t)=g\left(t+\bar{q}_{2}\right)$ and where $\bar{q}_{2}=\left(\mathbf{z}_{2}-\boldsymbol{\mu}_{2}\right)^{H} \boldsymbol{\Sigma}_{22}^{\#}\left(\mathbf{z}_{2}-\boldsymbol{\mu}_{2}\right)$.

\section{Singular non-circular complex normal distribution}

This section derives explicit expression of the p.d.f. for a singular NC-CN distribution. Let us first remind the reader that the non-singular NC-CN distribution was introduced in $[5,14,22]$, which can be viewed as a class of non-singular NC-CES distributions [11]. The non-singular NC-CN distribution has been recently widely used in various statistical signal processing applications such as: DOA methods [16-18], blind source separation methods [19-21], signal detection methods $[15,36,37]$, etc. Also Cramér-Rao performance bounds based on non-singular NC-CN distribution have been proposed for DOA estimation in [38] and source separation in [39]. Since 
non-singular NC-CN distribution is a member of non-singular NC-CES distributions, it follows that the p.d.f. of non-singular NC-CN distribution given below can be obtained from (2) by letting the density generator $g(t)$ equal to $g(t)=\exp (-t)$, which gives $c_{m, g}=\pi^{-m}$ and $\boldsymbol{\mu}=\mathrm{E}(\mathbf{z})$, the hermitian covariance matrix $\mathbf{R} \stackrel{\text { def }}{=} \operatorname{Cov}(\mathbf{z})=\boldsymbol{\Sigma}$ and the complex pseudocovariance matrix $\mathbf{R}^{\prime} \stackrel{\text { def }}{=} \operatorname{pcov}(\mathbf{z})=\boldsymbol{\Omega}$ exist.

Definition 1 A r.v. $\mathbf{z} \in \mathbb{C}^{m}$ has a non-singular $N C$-CN distribution (denoted $\mathbf{z} \sim \mathbb{C N}_{m}\left(\boldsymbol{\mu}, \mathbf{R}, \mathbf{R}^{\prime}\right)$ ) if its p.d.f. is of the form

$$
p(\mathbf{z})=(\pi)^{-m}(\operatorname{det}(\tilde{\mathbf{R}}))^{-1 / 2} \exp (-Q(\mathbf{z})),
$$

where $\tilde{\mathbf{R}} \stackrel{\text { def }}{=}\left(\begin{array}{cc}\mathbf{R} & \mathbf{R}^{\prime} \\ \mathbf{R}^{\prime *} & \mathbf{R}^{*}\end{array}\right) \in \mathbb{C}^{2 m \times 2 m}$ is assumed positive definite and $Q(\mathbf{z})$ is a quadratic form $Q(\mathbf{z}) \stackrel{\text { def }}{=}$ $\frac{1}{2}(\tilde{\mathbf{z}}-\tilde{\boldsymbol{\mu}})^{H} \tilde{\mathbf{R}}^{-1}(\tilde{\mathbf{z}}-\tilde{\boldsymbol{\mu}})$ where $\tilde{\mathbf{z}} \stackrel{\text { def }}{=}\left(\mathbf{z}^{H} \mathbf{z}^{T}\right)^{H}$ and $\tilde{\boldsymbol{\mu}} \stackrel{\text { def }}{=}$ $\left(\begin{array}{ll}\boldsymbol{\mu}^{H} & \boldsymbol{\mu}^{T}\end{array}\right)^{H}$.

In the special case where the pseudo-covariance matrix $\mathbf{R}^{\prime}=$ $\mathrm{O},(21)$ reduces to the following p.d.f. of non-singular $\mathrm{C}-\mathrm{CN}$ distribution,

$$
p(\mathbf{z})=\pi^{-m} \operatorname{det}\left(\mathbf{R}^{-1}\right) \exp (-Q(\mathbf{z})),
$$

where $Q(\mathbf{z}) \stackrel{\text { def }}{=}(\mathbf{z}-\boldsymbol{\mu})^{H} \mathbf{R}^{-1}(\mathbf{z}-\boldsymbol{\mu})$. Thus, non-singular C$\mathrm{CN}$ distribution can be seen as a special case of non-singular NC-CN distribution.

Recall that the matrix $\tilde{\mathbf{R}}$ is positive definite if and only if $\mathbf{R}$ and its schur complement $\mathbf{R}_{s}=\mathbf{R}-\mathbf{R}^{\prime} \mathbf{R}^{-*} \mathbf{R}^{\prime *}$ are definite positive [14]. However if these conditions are not met, $\tilde{\mathbf{R}}$ is a singular matrix and therefore the p.d.f. (21) does not exist. The following result gives the p.d.f. of singular NC-CN distribution which is obtained from result 2 by replacing $g(t)$ in (8) by $g(t)=\exp (-t)$, which gives $c_{m, g}=\pi^{-m}$ and $\boldsymbol{\Sigma}=\mathbf{R}$, $\mathbf{\Omega}=\mathbf{R}^{\prime}$.

Result 6 Let $\mathbf{z} \sim \mathbb{C N}_{m}\left(\boldsymbol{\mu}, \mathbf{R}, \mathbf{R}^{\prime}\right)$ with $\mathbf{R} \in \mathbb{C}^{m \times m}$ being a singular hermitian covariance matrix with $\operatorname{rank}(\mathbf{R})=$ $r<m$ and $\mathbf{R}^{\prime} \in \mathbb{C}^{m \times m}$ being a complex symmetric pseudocovariance matrix with $\operatorname{rank}\left(\mathbf{R}^{\prime}\right)=p$. In such case, the p.d.f. of a singular NC-CN distributed r.v. $\mathbf{z}$ is given by

$$
p(\mathbf{z})=\pi^{-r} c_{\lambda, \kappa}^{r, p} \exp (-Q(\mathbf{z}))
$$

and

$$
\mathbf{z}-\boldsymbol{\mu} \in(\operatorname{Ker} \mathbf{R})^{\perp} \text { w.p.1, }
$$

where $Q(\mathbf{z})$ is a quadratic form $Q(\mathbf{z}) \stackrel{\text { def }}{=} \frac{1}{2}(\tilde{\mathbf{z}}-\tilde{\boldsymbol{\mu}})^{H} \tilde{\mathbf{R}}^{\#}(\tilde{\mathbf{z}}-$ $\tilde{\boldsymbol{\mu}})$ and $c_{\lambda, \kappa}^{r, p} \stackrel{\text { def }}{=}\left(\prod_{k=1}^{r} \lambda_{k}\right)^{-1}\left(\prod_{l=1}^{p}\left(1-\left|\kappa_{l}\right|^{2}\right)\right)^{-\frac{1}{2}} \cdot \quad A$ singular NC-CN distributed r.v. will be denoted as $\mathbf{z} \sim$ $\mathbb{C} \mathcal{N}_{m}^{r}\left(\boldsymbol{\mu}, \mathbf{R}, \mathbf{R}^{\prime}\right)$.

Note that when $\mathbf{R}^{\prime}=\mathbf{O}$, the p.d.f. (23)-(24) of singular NC$\mathrm{CN}$ distribution reduces to the p.d.f. of singular C-CN distribution, which is given by the following result.
Result 7 Let $\mathbf{z} \sim \mathbb{C N}_{m}^{r}(\boldsymbol{\mu}, \mathbf{R}, \mathbf{O}) \equiv \mathbb{C N}_{m}^{r}(\boldsymbol{\mu}, \mathbf{R})$ where $\boldsymbol{\mu} \stackrel{\text { def }}{=} \mathrm{E}(\mathbf{z})$, with $\boldsymbol{\mu} \in \mathbb{C}^{m}, \mathbf{R} \in \mathbb{C}^{m \times m}$ and $\operatorname{rank}(\mathbf{R})=r<$ m. In such case, the p.d.f. of $\mathbf{z}$ is given by

$$
p(\mathbf{z})=\pi^{-r} c_{\lambda}^{r} \exp (-Q(\mathbf{z}))
$$

and

$$
\mathbf{z}-\boldsymbol{\mu} \in(\operatorname{Ker} \mathbf{R})^{\perp} \text { w.p.1. }
$$

where $c_{\lambda}^{r} \stackrel{\text { def }}{=} \prod_{k=1}^{r} \lambda_{k}^{-1}$ and $Q(\mathbf{z})=(\mathbf{z}-\boldsymbol{\mu})^{H} \mathbf{R}^{\#}(\mathbf{z}-\boldsymbol{\mu})$.

Remark 2 Note that the c.f. of singular $N C$-CN distributed r.v. $\mathbf{z} \sim \mathbb{C N}_{m}^{r}\left(\boldsymbol{\mu}, \mathbf{R}, \mathbf{R}^{\prime}\right)$ always exists and identical to the c.f. of non-singular $\mathrm{NC}-\mathrm{CN}$ distribution given by [14]

$$
\Phi(\mathbf{z})=\exp \left\{j \Re\left(\mathbf{z}^{H} \boldsymbol{\mu}\right)-\frac{1}{4}\left[\mathbf{z}^{H} \mathbf{R} \mathbf{z}+\Re\left(\mathbf{z}^{H} \mathbf{R}^{\prime} \mathbf{z}^{*}\right)\right]\right\} .
$$

It follows from result 5 that the p.d.f. of conditional distribution of two singular NC-CN distritbuted r.v.'s are summarized as the following result.

Result 8 Assume $\mathbf{y}_{1} \sim \mathbb{C N}_{d}^{r_{1}}\left(\boldsymbol{\mu}_{1}, \mathbf{R}_{11}, \mathbf{R}_{11}^{\prime}\right)$ and $\mathbf{y}_{2} \sim$ $\mathbb{C N}_{n}^{r_{2}}\left(\boldsymbol{\mu}_{2}, \mathbf{R}_{22}, \mathbf{R}_{22}^{\prime}\right)$. Let $\mathbf{R}_{12} \stackrel{\text { def }}{=} \mathrm{E}\left(\mathbf{y}_{1} \mathbf{y}_{2}^{H}\right)$ and $\mathbf{R}_{12}^{\prime} \stackrel{\text { def }}{=}$ $\mathrm{E}\left(\mathbf{y}_{1} \mathbf{y}_{2}^{T}\right)$. Then $\mathbf{y}_{1} \mid \mathbf{y}_{2} \sim \mathbb{C N}_{d}^{r_{1.2}}\left(\boldsymbol{\mu}_{y_{1} \mid y_{2}}, \mathbf{R}_{11.2}, \mathbf{R}_{11.2}^{\prime}\right)$ with

$$
\begin{aligned}
\boldsymbol{\mu}_{y_{1} \mid y_{2}} & =\boldsymbol{\mu}_{1}+\tilde{\mathbf{E}}\left(\mathbf{y}_{2}-\boldsymbol{\mu}_{2}\right)+\tilde{\mathbf{F}}\left(\mathbf{y}_{2}^{*}-\boldsymbol{\mu}_{2}^{*}\right), \\
\mathbf{R}_{11.2} & =\mathbf{R}_{11}-\tilde{\mathbf{E}} \mathbf{R}_{12}^{H}-\tilde{\mathbf{F}} \mathbf{R}_{12}^{\prime H} \\
\mathbf{R}_{11.2}^{\prime} & =\mathbf{R}_{11}^{\prime}-\tilde{\mathbf{E}} \mathbf{R}_{12}^{\prime T}-\tilde{\mathbf{F}} \mathbf{R}_{12}^{T} .
\end{aligned}
$$

where

$$
\begin{gathered}
\tilde{\mathbf{E}} \stackrel{\text { def }}{=}\left(\mathbf{R}_{12}-\mathbf{R}_{12}^{\prime} \mathbf{R}_{22}^{* \#} \mathbf{R}_{22}^{\prime H}\right) \mathbf{P}_{y_{2}}^{* \#}, \\
\tilde{\mathbf{F}} \stackrel{\text { def }}{=}\left(\mathbf{R}_{12}^{\prime}-\mathbf{R}_{12} \mathbf{R}_{22}^{\#} \mathbf{R}_{22}^{\prime}\right) \mathbf{P}_{y_{2}}^{\#},
\end{gathered}
$$

where $\mathbf{P}_{y_{2}} \stackrel{\text { def }}{=} \mathbf{R}_{22}^{*}-\mathbf{R}_{22}^{\prime H} \mathbf{R}_{22}^{\#} \mathbf{R}_{22}^{\prime}$, and $r_{1.2}=\operatorname{rank}\left(\mathbf{R}_{11.2}\right)$.

For the circular case which is characterized by all the matrices $\mathbf{R}_{x}^{\prime}$ being zero, result 8 reduces to the following corollary.

Corollary 3 Assume that $\mathbf{y}_{1} \sim \mathbb{C N}_{d}^{r_{1}}\left(\boldsymbol{\mu}_{1}, \mathbf{R}_{11}, \mathbf{O}\right)$ and $\mathbf{y}_{2} \sim$ $\mathbb{C N}_{n}^{r_{2}}\left(\boldsymbol{\mu}_{2}, \mathbf{R}_{22}, \mathbf{O}\right)$. Then $\mathbf{y}_{1} \mid \mathbf{y}_{2} \sim \mathbb{C N}_{d}^{r_{1.2}}\left(\boldsymbol{\mu}_{y_{1} \mid y_{2}}, \mathbf{R}_{1.2}\right)$ where $r_{1.2}=\operatorname{rank}\left(\mathbf{R}_{1.2}\right)$ with

$$
\begin{aligned}
\boldsymbol{\mu}_{y_{1} \mid y_{2}} & =\boldsymbol{\mu}_{1}+\mathbf{R}_{12} \mathbf{R}_{22}^{\#}\left(\mathbf{y}_{2}-\boldsymbol{\mu}_{2}\right), \\
\mathbf{R}_{1.2} & =\mathbf{R}_{11}-\mathbf{R}_{12} \mathbf{R}_{22}^{\#} \mathbf{R}_{12}^{H}
\end{aligned}
$$

\section{Singular circular and non-circular Compound- Gaussian Distributions}

Non-singular C-CCG distributions presented in [10] under the assumption of non-singular scatter matrix $\boldsymbol{\Sigma} \in \mathcal{C}^{m \times m}$ (i.e., $\operatorname{rank}(\boldsymbol{\Sigma})=m$ ), represent an important subclass of nonsingular C-CES distributions. The non-singular C-CCG distributions are widely employed in radar signal processing to describe the heavy-tailed clutter process as a product of two independent random processes 'texture' and 'speckle'. More 
precisely, a r.v. $\mathbf{z}$ has a non-singular C-CCG distribution if it admits a C-CCG-representation

$$
\mathbf{z}={ }_{d} \boldsymbol{\mu}+\sqrt{\tau} \mathbf{n}
$$

where $\tau$ is a positive real r.v. with p.d.f. $f_{\tau}$, called as texture, independent of $\mathbf{n} \sim \mathbb{C N}_{m}(\mathbf{0}, \boldsymbol{\Sigma})$, called as speckle. The p.d.f.'s of non-singular C-CCG-distributions are given by

$$
\begin{aligned}
p(\mathbf{z}) & =\pi^{-m}(\operatorname{det}(\boldsymbol{\Sigma}))^{-1} \int_{0}^{\infty} \tau^{-m} \exp (-q(\mathbf{z}) / \tau) d F_{\tau}(\tau) \\
& =\pi^{-m}(\operatorname{det}(\boldsymbol{\Sigma}))^{-1} \int_{0}^{\infty} \tau^{-m} \exp (-q(\mathbf{z}) / \tau) f_{\tau}(\tau)(2 \boldsymbol{2} 7,)
\end{aligned}
$$

where $q(\mathbf{z})=(\mathbf{z}-\boldsymbol{\mu})^{H} \boldsymbol{\Sigma}^{-1}(\mathbf{z}-\boldsymbol{\mu})$ and $f_{\tau}(\tau)=\partial F_{\tau}(\tau) / \partial \tau$. Note that the p.d.f (27) can always be written in the form (5) with a density generator $g(t) \propto \int_{0}^{\infty} \tau^{-m} \exp (-t / \tau) f_{\tau}(\tau) d \tau$. A non-singular C-CCG distributed r.v. will be denoted as $\mathbf{z} \sim$ $\mathbb{C N}_{m}(\boldsymbol{\mu}, \boldsymbol{\Sigma})$. Different choices of distribution for $f_{\tau}($.$) lead$ to some well-known examples of CCG-distributions such as $t$-distribution and $K$-distribution presented in the next section.

Note that the p.d.f. of singular NC-CN distributions presented in section 3 exist on a subspace. Given the above definition of non-singular C-CCG distributions, the p.d.f. of singular NC-CCG distributions also exist on a subspace and can be defined as follows.

Definition 2 A r.v. $\mathbf{z} \in \mathbb{C}^{m}$ is said to have a singular NC-CCG distribution if it admits a NC-CCG representation

$$
\mathbf{z}={ }_{d} \boldsymbol{\mu}+\sqrt{\tau} \mathbf{n},
$$

where $\tau$ is a r.v. defined above and independent of $\mathbf{n}$ follows the singular NC-CN distribution $\mathbb{C N}_{m}^{r}(\mathbf{0}, \boldsymbol{\Sigma}, \boldsymbol{\Omega})$. Also, the p.d.f.'s of singular NC-CCG distributions are given by

$$
p(\mathbf{z})=\pi^{-r} c_{\lambda, \kappa}^{r, p} \int_{0}^{\infty} \tau^{-r} \exp (-q(\mathbf{z}) / \tau) f_{\tau}(\tau) d \tau
$$

and

$$
\mathbf{z}-\boldsymbol{\mu} \in(\operatorname{Ker} \boldsymbol{\Sigma})^{\perp} \text { w.p.1, }
$$

where $c_{\lambda, \kappa}^{r, p}$ and the quadratic form $q(\mathbf{z})$ are defined in result 2. A singular NC-CCG distributed r.v. will be denoted as $\mathbf{z} \sim$ $\mathbb{C N}_{m}^{r}(\boldsymbol{\mu}, \boldsymbol{\Sigma}, \boldsymbol{\Omega})$. In the special case when $\boldsymbol{\Omega}=\mathbf{O},(29)$ reduces to the following expression of the p.d.f. of singular C-CCG distributions (denoted as $\mathbf{z} \sim \mathbb{C N}_{m}^{r}(\boldsymbol{\mu}, \boldsymbol{\Sigma})$ )

$$
p(\mathbf{z})=\pi^{-r} c_{\lambda}^{r} \int_{0}^{\infty} \tau^{-r} \exp (-q(\mathbf{z}) / \tau) f_{\tau}(\tau) d \tau,
$$

where $q(\mathbf{z})$ and $c_{\lambda}^{r}$ are defined in result 4 .

If $\tau$ has a finite second-order moments (i.e., $\mathrm{E}(\tau)<\infty$ ), the mean and the second-order moments of $\mathbf{z}$ exist. It also follows that if $\mathrm{E}(\tau)=1$, the scatter matrix $\Sigma$ and pseudo-scatter matrix $\Omega$ are, respectively, exactly equal to the covariance matrix $\operatorname{Cov}(\mathbf{z})=\mathrm{E}(\tau) \operatorname{Cov}(\mathbf{n})=\boldsymbol{\Sigma}$ and pseudo covariance matrix $\operatorname{pcov}(\mathbf{z})=\mathrm{E}(\tau) \operatorname{pcov}(\mathbf{n})=\boldsymbol{\Omega}$. The following result proved in Appendix E gives the stochastic representation of the quadratic form in singular NC-CES distributions.
Result 9 Let $\mathbf{z}=\mathbf{z}_{r}+j \mathbf{z}_{i} \sim \mathbb{C N}_{m}(\boldsymbol{\mu}, \boldsymbol{\Sigma}, \boldsymbol{\Omega})$ and $\tilde{\mathbf{Q}} \in \mathbb{C}^{2 m \times 2 m}$ be a hermitian matrix partitioned as $\tilde{\mathbf{Q}} \stackrel{\text { def }}{=}$ $\left(\begin{array}{cc}\mathbf{Q} & \mathbf{Q}^{\prime} \\ \mathbf{Q}^{\prime *} & \mathbf{Q}^{*}\end{array}\right)$ where $\mathbf{Q} \in \mathbb{C}^{m \times m}$ is a hermitian matrix and $\mathbf{Q}^{\prime} \in \mathbb{C}^{m \times m}$ is a symmetric complex matrix. Then the stochastic representation of the quadratic form $\tilde{\mathbf{z}}_{0}^{H} \tilde{\mathbf{Q}}_{0} \tilde{\mathbf{z}}_{0}$ where $\tilde{\mathbf{z}}_{0} \stackrel{\text { def }}{=} \tilde{\mathbf{z}}-\tilde{\boldsymbol{\mu}}$ is given by

$$
\tilde{\mathbf{z}}_{0}^{H} \tilde{\mathbf{Q}} \tilde{\mathbf{z}}_{0}={ }_{d} \tau \sum_{l=1}^{q} \lambda_{l} \chi_{1}^{2}(l),
$$

where the $\chi_{1}^{2}(l)$ are independent central Chi-square random variables with one degree of freedom. The $\lambda_{l}$ are nonzero eigenvalues of the matrix $\tilde{\boldsymbol{\Gamma}} \tilde{\mathbf{Q}}$ of rank $q$ where $\tilde{\boldsymbol{\Gamma}}$ is defined in (2).

For the special case of non-singular C-CCG distributions where $\Omega=\mathbf{O}$, assume that $\mathbf{Q}^{\prime}=\mathbf{O}$, result (9) reduces to the following corollary:

Corollary 4 Let $\mathbf{z} \sim \mathbb{C N}_{m}(\boldsymbol{\mu}, \boldsymbol{\Sigma})$ and $\mathbf{Q} \in \mathbb{C}^{m \times m}$ be a hermitian matrix. Then the stochastic representation of the quadratic form $\mathbf{z}_{0}^{H} \mathbf{Q} \mathbf{z}_{0}$ where $\mathbf{z}_{0} \stackrel{\text { def }}{=} \mathbf{z}-\boldsymbol{\mu}$ is given by

$$
\mathbf{z}_{0}^{H} \mathbf{Q} \mathbf{z}_{0}=\frac{1}{2} \tilde{\mathbf{z}}_{0}^{H} \tilde{\mathbf{Q}} \tilde{\mathbf{z}}_{0}={ }_{d} \frac{\tau}{2} \sum_{l=1}^{q_{c}} \lambda_{l} \chi_{2}^{2}(l),
$$

where the $\lambda_{l}$ are nonzero eigenvalues of the matrix $\mathbf{\Sigma} \mathbf{Q}$ of rank $q_{c}$.

\section{Examples of singular NC-CES distributions}

Based on the results of section 2, and similar to the nonsingular case $(\boldsymbol{\Sigma}>0)[5,10,11]$, we provide explicit expressions for the p.d.f.'s of three subclasses of CES distributions,i.e., complex $K$-distribution, complex $t$-distribution and complex generalized Gaussian (CGG) distribution, under the assumption of singular scatter matrix $(\operatorname{rank}(\boldsymbol{\Sigma})=r)$. These subclasses of distributions can be distinguished from each other only by their functional form of the density generator $g($.$) as shown below.$

\section{Example 1: Singular non-circular complex $K$-distribution}

It follows from result 2 that the singular non-circular complex $K$-distribution can exist on subspace and its p.d.f. is given by the following definition.

Definition 3 A r.v. $\mathbf{z} \in \mathbb{C}^{m}$ is said to have a singular noncircular complex $K$-distribution with parameters $\boldsymbol{\mu} \in \mathbb{C}^{m}$; $\boldsymbol{\Omega} \in \mathbb{C}^{m \times m}$ and $\operatorname{rank}(\boldsymbol{\Omega})=p ; \boldsymbol{\Sigma} \in \mathbb{C}^{m \times m}, \boldsymbol{\Sigma} \geq 0$ and $\operatorname{rank}(\boldsymbol{\Sigma})=r$ if its p.d.f. is of the form

$$
f_{K}(\mathbf{z})=c_{r, g_{K}} c_{\lambda, \kappa}^{r, p} g_{K}(q(\mathbf{z})),
$$

and

$$
\mathbf{z}-\boldsymbol{\mu} \in(\operatorname{Ker} \boldsymbol{\Sigma})^{\perp} \text { w.p.l, }
$$

where the quadratic form $q(\mathbf{z})$ defined in result $2, c_{r, g_{K}}=$ $2 \nu^{(r+\nu) / 2} /\left[\Gamma(\nu) \pi^{r}\right]$ is a normalizing constant, $g_{K}($.$) is the$ density generator given by $g_{K}(t)=t^{(\nu-r) / 2} K_{\nu-r}(2 \sqrt{\nu t}), \nu$ 
is the shape parameter which controls the shape of complex $K$-distribution, $K_{\ell}($.$) denotes the modified Bessel function of$ the second kind of order $\ell$. The singular non-circular complex $K$-distribution is a class of singular NC-CCG distribution and it has the singular NC-CCG representation (28) where the unit mean texture variable $\tau$ follows a gamma distribution with shape parameter $\nu>0$ and scale parameter $1 / \nu$, denoted $\tau \sim \operatorname{Gamma}(\tau, 1 / \tau)$. A singular non-circular complex $K$ distribution will be denoted by $\mathbb{C} K_{m, \nu}^{r}(\mu, \boldsymbol{\Sigma}, \boldsymbol{\Omega})$.

\section{Example 2: Singular non-circular complex t-distribution}

It follows also from result 2 that the singular non-circular complex $t$-distribution can exist on subspace and its p.d.f. is given by the following definition.

Definition 4 A r.v. $\mathbf{z} \in \mathbb{C}^{m}$ is said to have a singular noncircular complex $t$-distribution with parameters $\boldsymbol{\mu} \in \mathbb{C}^{m}$; $\boldsymbol{\Omega} \in \mathbb{C}^{m \times m}$ and $\operatorname{rank}(\boldsymbol{\Omega})=p ; \boldsymbol{\Sigma} \in \mathbb{C}^{m \times m}, \boldsymbol{\Sigma} \geq 0$ and $\operatorname{rank}(\boldsymbol{\Sigma})=r$ if its p.d.f. is of the form

$$
f_{T}(\mathbf{z})=c_{r, g_{T}} c_{\lambda, \kappa}^{r, p} g_{T}(q(\mathbf{z})),
$$

and

$$
\mathbf{z}-\boldsymbol{\mu} \in(\operatorname{Ker} \boldsymbol{\Sigma})^{\perp} \text { w.p.l, }
$$

where the quadratic form $q(\mathbf{z})$ defined in result 2, $c_{r, g_{T}}=2^{r} \Gamma\left(\frac{2 r+\nu}{2}\right) /\left[(\pi \nu)^{r} \Gamma\left(\frac{\nu}{2}\right)\right]$ is a normalizing constant and $g_{T}($.$) is the density generator given by g_{T}(t)=$ $\left(1+\frac{2 t}{\nu}\right)^{-(2 r+\nu) / 2}$ with $\nu$ degrees of freedom $(2<\nu<\infty)$. If $\nu=1$, the case is called the complex Cauchy distribution, and if $\nu$ goes to $\infty$, it yields the singular $N C-C N$ distribution. The singular non-circular complex $t$-distribution is also a class of singular NC-CCG distributions and it has the singular NCCCG-representation (28) where the texture r.v. $\tau$ distributes as $\tau={ }_{d}(\nu-2) / \chi_{\nu}^{2}\left(\right.$ where $\left.\chi_{\nu}^{2}=\operatorname{Gamma}(\tau / 2,2 / \tau)\right)$. A singular non-circular complex $t$-distribution will be denoted by $\mathbb{C} t_{m, \nu}^{r}(\mu, \boldsymbol{\Sigma}, \boldsymbol{\Omega})$.

\section{Example 3: Singular NC-CGG distribution}

Similarly, the following definition provides the p.d.f. of singular NC-CGG distribution:

Definition 5 A r.v. $\mathbf{z} \in \mathbb{C}^{m}$ is said to have a singular noncircular complex $G G(N C-C G G)$ distribution with exponent $s>0$ and scale $b>0$ and parameters $\boldsymbol{\mu} \in \mathbb{C}^{m} ; \boldsymbol{\Omega} \in \mathbb{C}^{m \times m}$ and $\operatorname{rank}(\boldsymbol{\Omega})=p ; \boldsymbol{\Sigma} \in \mathbb{C}^{m \times m}, \boldsymbol{\Sigma} \geq 0$ and $\operatorname{rank}(\boldsymbol{\Sigma})=r$ if its p.d.f. is of the form

$$
f_{G}(\mathbf{z})=c_{r, g_{G}} c_{\lambda, \kappa}^{r, p} g_{G}\left(q_{s}^{c}(\mathbf{z})\right),
$$

and

$$
\mathbf{z}-\boldsymbol{\mu} \in(\operatorname{Ker} \boldsymbol{\Sigma})^{\perp} \text { w.p.l, }
$$

where the quadratic form $q(\mathbf{z})$ is defined in result 2 and $g_{G}($. is the density generator given by $g_{G}(t)=\exp \left(-t^{s} / b\right)$, which gives $c_{r, g_{G}}=s \Gamma(r) b^{-r / s} /\left[\pi^{r} \Gamma(r / s)\right]$ as the value of the normalizing constant. Note that for this singular NC-CGG distribution, the 2 nd-order modular variate $\mathcal{Q}=\mathcal{R}^{2}$ is distributed as $\mathcal{Q}={ }_{d} G^{1 / s}$ where $G$ is a r.v. distributed according to a Gamma distribution with shape $\mathrm{m} / \mathrm{s}$ and scale $b$. A singular NC-CGG distribution will be denoted by $\mathbb{C} G G_{m, s, b}^{r}(\mu, \boldsymbol{\Sigma}, \boldsymbol{\Omega})$.
It follows from definitions 3-5 that the singular circular complex $K$-distribution, the singular circular complex $t$ distribution and the singular circular CGG (C-CGG) distribution are obtained if $\boldsymbol{\Omega}=\mathbf{O}$ and thus $\mathbb{C} K_{m, \nu}^{r}(\mu, \boldsymbol{\Sigma}) \equiv$ $\mathbb{C} K_{m, \nu}^{r}(\mu, \boldsymbol{\Sigma}, \mathbf{O}), \mathbb{C} t_{m, \nu}^{r}(\mu, \boldsymbol{\Sigma}) \equiv \mathbb{C} t_{m, \nu}^{r}(\mu, \boldsymbol{\Sigma}, \mathbf{O})$ and $\mathbb{C} G G_{m, s, b}^{r} C(\mu, \boldsymbol{\Sigma}) \equiv \mathbb{C} G G_{m, s, b}^{r}(\mu, \boldsymbol{\Sigma}, \mathbf{O})$.

\section{Singular widely linear mean square estimation}

This section extends the results on linear or widely linear minimum mean-square error (LMMSE or WLMMSE) estimation of a signal from non-singular distributed measurement data vector [28] to the case of estimating a signal from singular distributed measurement data vector.

Let $\mathbf{z}_{1} \sim \mathrm{EC}_{d}^{r_{1}}\left(\mathbf{0}, \boldsymbol{\Sigma}_{11}, \boldsymbol{\Omega}_{11}, g\right)$ be a singular NC-CES distributed r.v. that need to be estimated from a singular NCCES distributed r.v. $\mathbf{z}_{2} \sim \operatorname{EC}_{n}^{r_{2}}\left(\mathbf{0}, \boldsymbol{\Sigma}_{22}, \boldsymbol{\Omega}_{22}, g\right)$, as introduced in result 5 . As usual $\mathbf{z}_{1}$ is considered as signal or source and $\mathbf{z}_{2}$ as the measurement or observation. We remind the reader here that the scatter matrix $\boldsymbol{\Sigma}_{22}$ is singular and that $\delta_{n, g}<\infty$ such that the covariance matrix $\operatorname{Cov}\left(\mathbf{z}_{2}\right)=\boldsymbol{\Sigma}_{22}$ and the pseudo-covariance matrix $\operatorname{pcov}(\mathbf{z})=\Omega_{22}$ exist. Let $\boldsymbol{\Sigma}_{12}$ and $\boldsymbol{\Omega}_{12}$ be two matrices defined as $\boldsymbol{\Sigma}_{12} \stackrel{\text { def }}{=} \mathrm{E}\left(\mathbf{z}_{1} \mathbf{z}_{2}^{H}\right)$ and $\boldsymbol{\Omega}_{12} \stackrel{\text { def }}{=} \mathrm{E}\left(\mathbf{z}_{1} \mathbf{z}_{2}^{T}\right)$.

It follows from result 5 that the conditional mean $\mathbf{m}\left(\mathbf{z}_{2}\right) \stackrel{\text { def }}{=}$ $\mathrm{E}\left(\mathbf{z}_{1} \mid \mathbf{z}_{2}\right)$ can be expressed as a function of $\mathbf{z}_{2}$ and $\mathbf{z}_{2}^{*}$ as follows:

$$
\mathbf{m}\left(\mathbf{z}_{2}\right)=\mathbf{E} \mathbf{z}_{2}+\mathbf{F} \mathbf{z}_{2}^{*}
$$

where $\mathbf{E}$ and $\mathbf{F}$ are two matrices defined in result 5, both of which depend on the pseudo-inverse operator. It is clear that $\mathbf{m}\left(\mathbf{z}_{2}\right)$ is singular widely linear (SWL) in $\mathbf{z}_{2}$. Note that the estimator $\mathbf{m}\left(\mathbf{z}_{2}\right)$ is called here the singular widely linear minimum mean-squared error (SWLMMSE) estimator of $\mathbf{z}_{1}$ from $\mathbf{z}_{2}$. The error covariance matrix of the SWLMMSE estimator is the covariance matrix of the conditional distribution of $\mathbf{z}_{1}$ given $\mathbf{z}_{2}$, and it follows from result 5 that it is given by

$$
\boldsymbol{\Sigma}_{e_{n c}}=\boldsymbol{\Sigma}_{11}-\mathbf{E} \boldsymbol{\Sigma}_{12}^{H}-\mathbf{F} \boldsymbol{\Omega}_{12}^{H} .
$$

The following subsections consider two cases when $\mathbf{z}_{2}$ is a circular r.v. and $\mathbf{z}_{1}$ is a real r.v.

\subsection{Circular case}

If $\mathbf{z}_{1}$ and $\mathbf{z}_{2}$ are cross-circular r.v.'s, $\boldsymbol{\Omega}_{12}=\mathbf{O}$, and $\mathbf{z}_{2}$ is a circular r.v., $\Omega_{22}=\mathbf{O}$, it follows that $\mathbf{F}=\mathbf{O}$ in (34), and therefore the SWLMMSE estimator reduces to the following singular linear minimum mean-squared error (SLMMSE) estimator

$$
\mathbf{m}^{\prime}\left(\mathbf{z}_{2}\right)=\boldsymbol{\Sigma}_{12} \boldsymbol{\Sigma}_{22}^{\#} \mathbf{z}_{2}
$$

Similarly, the error covariance matrix of the SLMMSE estimator is the covariance matrix of the conditional distribution of $\mathbf{z}_{1}$ given $\mathbf{z}_{2}$, and it follows from corollary 2 that it is given by

$$
\boldsymbol{\Sigma}_{e_{c}}=\boldsymbol{\Sigma}_{11}-\boldsymbol{\Sigma}_{12} \boldsymbol{\Sigma}_{22}^{\#} \boldsymbol{\Sigma}_{12}^{H} .
$$




\subsection{Real case}

If $\mathbf{z}_{1}$ is a real-valued parameter vector with singular CES distribution, it is singular NC-CES ditsributed r.v., and the application of SWLMMSE estimator is obvious. In this case, $\Omega_{12}=\Sigma_{12}^{*}$, and consequently from (34), the SWLMMSE estimator is of the form

$$
\mathbf{m}_{r}\left(\mathbf{z}_{2}\right)=2 \Re\left(\mathbf{E} \mathbf{z}_{2}\right) .
$$

Therefore, in this case the SWLMMSE estimator produces real-valued estimates, while the SLMMSE estimate is generally complex.

Similarly, it follows from (35) that the error covariance matrix takes the form

$$
\boldsymbol{\Sigma}_{e_{r}}=\boldsymbol{\Sigma}_{11}-\Re\left(\mathbf{E} \boldsymbol{\Sigma}_{12}^{H}\right) .
$$

\section{Application}

This section studies the problem of estimating the deterministic but unknown parameter vector of complex-valued linear model in the presence either of singular NC-CES or C-CES distributed error terms. After deriving the compact expressions of the maximum likelihood (ML) estimates of the parameters and their associated covariance matrices, we show that the associated residuals have singular NC-CES distributions.

Consider the complex-valued non-circular multivariate linear model

$$
\mathbf{z}=\mathbf{X} \boldsymbol{\alpha}+\varepsilon,
$$

where $\varepsilon, \mathbf{z} \in \mathbb{C}^{m}, \mathbf{X} \in \mathbb{C}^{m \times n}$ is a known matrix of full column rank $n$ and $\boldsymbol{\alpha} \in \mathbb{C}^{m}$ is an unknown deterministic vector parameter to be estimated. Assume $\varepsilon \sim \operatorname{EC}_{m}^{r}(\mathbf{0}, \boldsymbol{\Sigma}, \boldsymbol{\Omega}, g)$ such that $\mathbf{z} \sim \mathrm{EC}_{m}^{r}(\mathbf{X} \boldsymbol{\alpha}, \boldsymbol{\Sigma}, \boldsymbol{\Omega}, g), \boldsymbol{\Sigma}=\sigma_{\varepsilon}^{2} \boldsymbol{\Sigma}^{\prime}$ is singular hermitian matrix with $\operatorname{rank}(\boldsymbol{\Sigma})=r$ and $\boldsymbol{\Omega}=\sigma_{\varepsilon}^{2} \boldsymbol{\Omega}^{\prime}$ is complex symmetric matrix where $\sigma_{\varepsilon}^{2}$ is assumed unknown but $\boldsymbol{\Sigma}^{\prime}$ and $\boldsymbol{\Omega}^{\prime}$ are known. Since $\mathbf{z}$ is a non-circular complex r.v., the complexvalued linear model (38) in augmented form is

$$
\tilde{\mathbf{z}}=\tilde{\mathbf{X}} \tilde{\boldsymbol{\alpha}}+\tilde{\varepsilon},
$$

where $\tilde{\mathbf{z}} \stackrel{\text { def }}{=}\left(\begin{array}{ll}\mathbf{y}^{H} & \mathbf{y}^{T}\end{array}\right)^{H}, \tilde{\mathbf{X}} \stackrel{\text { def }}{=}\left(\begin{array}{cc}\mathbf{X} & \mathbf{O} \\ \mathbf{O} & \mathbf{X}^{*}\end{array}\right), \tilde{\boldsymbol{\alpha}} \stackrel{\text { def }}{=}$ $\left(\begin{array}{ll}\boldsymbol{\alpha}^{H} & \boldsymbol{\alpha}^{T}\end{array}\right)^{H}$ and $\tilde{\varepsilon} \stackrel{\text { def }}{=}\left(\begin{array}{ll}\varepsilon^{H} & \varepsilon^{T}\end{array}\right)^{H}$.

\subsection{Singular C-CES distributed error term}

For singular C-CES distributions, the matrix $\boldsymbol{\Omega}=\mathbf{O}$ and it follows that $\mathbf{z} \sim \mathrm{EC}_{m}^{r}(\mathbf{X} \boldsymbol{\alpha}, \boldsymbol{\Sigma}, g)$. For fixed $\sigma_{\varepsilon}^{2}$, the $\mathrm{ML}$ estimator of $\boldsymbol{\alpha}$, denoted $\hat{\boldsymbol{\alpha}}$, is values of $\alpha$ that maximizes the p.d.f. (12). Since the function $g($.$) is monotonically decreasing$ in $[0 \infty)$, it follows that maximizing the p.d.f. (12) with respect to $\boldsymbol{\alpha}$ is equivalent to maximizing the quadratic cost function

$$
q_{s}^{c}(\mathbf{z})=(\mathbf{z}-\mathbf{X} \boldsymbol{\alpha})^{H} \mathbf{\Sigma}^{\#}(\mathbf{z}-\mathbf{X} \boldsymbol{\alpha}) .
$$

Since $\mathbf{X}$ has full column rank and $\mathbf{X}^{H} \boldsymbol{\Sigma}^{\#} \mathbf{X}$ is non-singular matrix, the ML estimator $\hat{\boldsymbol{\alpha}}$ is given by

$$
\hat{\boldsymbol{\alpha}}=\left(\mathbf{X}^{H} \boldsymbol{\Sigma}^{\#} \mathbf{X}\right)^{-1} \mathbf{X}^{H} \boldsymbol{\Sigma}^{\#} \mathbf{z} .
$$

It is easy to verify that $\mathrm{E}(\hat{\boldsymbol{\alpha}})=\boldsymbol{\alpha}$ and $\hat{\boldsymbol{\alpha}}$ is unbiased with covariance matrix

$$
\begin{aligned}
\operatorname{Cov}(\hat{\boldsymbol{\alpha}})= & \left(\mathbf{X}^{H} \boldsymbol{\Sigma}^{-1} \mathbf{X}\right)^{\#} \mathbf{X}^{H} \boldsymbol{\Sigma}^{\#} \\
& \operatorname{Cov}(\mathbf{z}) \boldsymbol{\Sigma}^{\#} \mathbf{X}\left(\mathbf{X}^{H} \boldsymbol{\Sigma}^{\#} \mathbf{X}\right)^{-1} \\
= & c_{0}\left(\mathbf{X}^{H} \boldsymbol{\Sigma}^{\#} \mathbf{X}\right)^{-1}
\end{aligned}
$$

using $\operatorname{Cov}(\mathbf{z})=c_{0} \boldsymbol{\Sigma}$ and $\boldsymbol{\Sigma}^{\#} \boldsymbol{\Sigma} \boldsymbol{\Sigma}^{\#}=\boldsymbol{\Sigma}^{\#}$ [40] where $c_{0}$ is a positive real scalar. Given that ML estimator $\hat{\boldsymbol{\alpha}}$ is a linear transformation of multivariate non-singular C-CES distributed vector $\mathbf{z}$, the ML estimator is non-singular C-CES distributed

$$
\hat{\boldsymbol{\alpha}} \sim \mathrm{EC}_{n}\left(\boldsymbol{\alpha},\left(\mathbf{X}^{H} \boldsymbol{\Sigma}^{\#} \mathbf{X}\right)^{-1}, g\right) .
$$

The residuals vector can be defined as

$$
\mathbf{e}_{c} \stackrel{\text { def }}{=} \mathbf{z}-\mathbf{X} \hat{\boldsymbol{\alpha}}=\mathbf{H}_{c} \mathbf{z},
$$

where $\mathbf{H}_{c} \stackrel{\text { def }}{=} \mathbf{I}-\mathbf{H}$ is idempotent matrix and $\mathbf{H} \stackrel{\text { def }}{=}$ $\mathbf{X}\left(\mathbf{X}^{H} \boldsymbol{\Sigma}^{\prime \#} \mathbf{X}\right)^{-1} \mathbf{X}^{H} \boldsymbol{\Sigma}^{\prime \#}$. Note that, since $\mathbf{H}_{c}$ is singular with $\operatorname{rank}\left(\mathbf{H}_{c}\right)=m-n$ and $\mathbf{H}_{c} \mathbf{X}=\mathbf{O}$, it also follows that

$$
\mathbf{e}_{c} \sim \mathrm{EC}_{m}^{m-n}\left(\mathbf{0}, \sigma_{\varepsilon}^{2} \mathbf{H}_{c} \boldsymbol{\Sigma}^{\prime} \mathbf{H}_{c}^{H}, g\right)
$$

Hence by (44) we have

$$
\begin{aligned}
\mathrm{E}\left(\mathbf{e}_{c}^{H}\left(\mathbf{H}_{c} \boldsymbol{\Sigma}^{\prime} \mathbf{H}_{c}^{H}\right)^{\#} \mathbf{e}_{c}\right) & =\operatorname{Tr}\left(\left(\mathbf{H}_{c} \boldsymbol{\Sigma}^{\prime} \mathbf{H}_{c}^{H}\right)^{\#} \mathrm{E}\left(\mathbf{e}_{c} \mathbf{e}_{c}^{H}\right)\right) \\
& =\sigma_{\varepsilon}^{2} c_{0} \operatorname{Tr}\left(\left(\mathbf{H}_{c} \boldsymbol{\Sigma}^{\prime} \mathbf{H}_{c}^{H}\right)^{\#}\left(\mathbf{H}_{c} \boldsymbol{\Sigma}^{\prime} \mathbf{H}_{c}^{H}\right)\right) \\
& =\sigma_{\varepsilon}^{2} c_{0}(m-n),
\end{aligned}
$$

where $c_{0}$ is a constant defined in (42) which takes different values according to the choices of CES distributions. It follows that the following statistic $\hat{\sigma}_{\varepsilon}^{2}$ defined in (45) is an unbiased estimator of $\sigma_{\varepsilon}^{2}$

$$
\hat{\sigma}_{\varepsilon}^{2}=\frac{\mathbf{e}_{c}^{H}\left(\mathbf{H}_{c} \boldsymbol{\Sigma}^{\prime} \mathbf{H}_{c}^{H}\right)^{\#} \mathbf{e}_{c}}{c_{0}(m-n)} .
$$

Since the C-CCG distributions presented in section 4 form a subclass of the CES distributions, it follows from corollary 4 that, if $\boldsymbol{\varepsilon} \sim \mathbb{C N}_{m}^{r}(\mathbf{0}, \boldsymbol{\Sigma})$, the quadratic from $\frac{\mathbf{e}_{c}^{H}\left(\mathbf{H}_{c} \boldsymbol{\Sigma}^{\prime} \mathbf{H}_{c}^{H}\right)^{\#} \mathbf{e}_{c}}{\sigma_{\varepsilon}^{2}}$ has the following representation

$$
\frac{\mathbf{e}_{c}^{H}\left(\mathbf{H}_{c} \boldsymbol{\Sigma}^{\prime} \mathbf{H}_{c}^{H}\right)^{\#} \mathbf{e}_{c}}{\sigma_{\varepsilon}^{2}}={ }_{d} \frac{\tau}{2} \chi_{2 q_{c}}^{2},
$$

where $q_{c}=\operatorname{rank}\left(\mathbf{H}_{c} \boldsymbol{\Sigma}^{\prime} \mathbf{H}_{c}^{H}\right)=m-n$. Therefore, the statistic $\hat{\sigma}_{\varepsilon}^{2}$ in (45) remains unbiased estimator of $\sigma_{\varepsilon}^{2}$ where here $c_{0}=$ $\mathrm{E}(\tau)$.

\subsection{Singular NC-CES distributed error term}

The r.v. $\mathbf{z}$ is assumed to have singular NC-CES distribution, i.e., $\mathbf{z} \sim \mathrm{EC}_{m}^{r}(\mathbf{X} \boldsymbol{\alpha}, \boldsymbol{\Sigma}, \boldsymbol{\Omega}, g)$. Following the same reasoning as above, the ML estimator of $\tilde{\boldsymbol{\alpha}}$, denoted $\hat{\tilde{\boldsymbol{\alpha}}}$ for the model (39) is obtained as follows.

$$
\widehat{\tilde{\boldsymbol{\alpha}}}=\arg \min _{\tilde{\boldsymbol{\alpha}}} q_{s}^{n c}(\mathbf{z})=(\tilde{\mathbf{z}}-\tilde{\mathbf{X}} \tilde{\boldsymbol{\alpha}})^{H} \tilde{\boldsymbol{\Gamma}}^{\#}(\tilde{\mathbf{z}}-\tilde{\mathbf{X}} \tilde{\boldsymbol{\alpha}}),
$$


where $\tilde{\boldsymbol{\Gamma}}$ is defined in result 4 by $\tilde{\boldsymbol{\Gamma}} \stackrel{\text { def }}{=}\left(\begin{array}{cc}\boldsymbol{\Sigma} & \boldsymbol{\Omega} \\ \boldsymbol{\Omega}^{*} & \boldsymbol{\Sigma}^{*}\end{array}\right)$. The solution is given by

$$
\hat{\tilde{\boldsymbol{\alpha}}}=\left(\tilde{\mathbf{X}}^{H} \tilde{\boldsymbol{\Gamma}}^{\#} \tilde{\mathbf{X}}\right)^{-1} \tilde{\mathbf{X}}^{H} \tilde{\boldsymbol{\Gamma}}^{\#} \tilde{\mathbf{z}} .
$$

It is clear that this estimator is unbiased with covariance matrix

$$
\operatorname{Cov}(\hat{\tilde{\boldsymbol{\alpha}}})=c_{0}\left(\tilde{\mathbf{X}}^{H} \tilde{\boldsymbol{\Gamma}}^{\#} \tilde{\mathbf{X}}\right)^{-1},
$$

where $c_{0}$ is positive real valued scalar such that $\operatorname{Cov}(\mathbf{z})=$ $c_{0} \boldsymbol{\Sigma}$ and $\operatorname{pcov}(\mathbf{z})=c_{0} \boldsymbol{\Omega}$. It is easy to remark that for singular C-CES error where $\Omega=O$, the non-circular ML estimator (46) reduces to the circular ML estimator (41). Since $\tilde{\boldsymbol{\Gamma}}$ is $(2 m \times 2 m)$ structured block matrix, its MoorePenrose pseudo-inverse has the same structure and can be expressed using eigenvalue decomposition (57) as $\tilde{\boldsymbol{\Gamma}}^{\# \stackrel{\text { def }}{=}}$ $\left(\begin{array}{cc}\mathbf{G} & \mathbf{P} \\ \mathbf{P}^{*} & \mathbf{G}^{*}\end{array}\right)$ where $\mathbf{G} \stackrel{\text { def }}{=}\left(\boldsymbol{\Sigma}-\boldsymbol{\Omega} \boldsymbol{\Sigma}^{\# *} \boldsymbol{\Omega}^{*}\right)^{\#}$ and $\mathbf{P} \stackrel{\text { def }}{=}$ $-\boldsymbol{\Sigma}^{\#} \boldsymbol{\Omega} \mathrm{G}^{*}$ are hermitian and complex symmetric matrices, respectively. It follows that $\tilde{\mathbf{X}}^{H} \tilde{\boldsymbol{\Gamma}}^{\#} \tilde{\mathbf{X}}$ has the same structure and by using the matrix inversion lemma [40], its inverse is given by $\left(\tilde{\mathbf{X}}^{H} \tilde{\boldsymbol{\Gamma}}^{\#} \tilde{\mathbf{X}}\right)^{-1} \stackrel{\text { def }}{=}\left(\begin{array}{cc}\mathbf{K} & \mathbf{L} \\ \mathbf{L}^{*} & \mathbf{K}^{*}\end{array}\right)$ where $\mathbf{K} \stackrel{\text { def }}{=}\left(\mathbf{X}^{H} \mathbf{G X}-\left(\mathbf{X}^{H} \mathbf{P} \mathbf{X}^{*}\right)\left(\mathbf{X}^{T} \mathbf{G}^{*} \mathbf{X}^{*}\right)^{-1}\left(\mathbf{X}^{T} \mathbf{P}^{*} \mathbf{X}\right)\right)^{-1}$ and $\mathbf{L} \stackrel{\text { def }}{=}-\left(\mathbf{X}^{H} \mathbf{G X}\right)^{-1}\left(\mathbf{X}^{H} \mathbf{P} \mathbf{X}^{*}\right) \mathbf{K}^{*}$ are hermitian and complex symmetric matrices, respectively. Therefore, from (46) and after simple algebraic manipulations, the non-circular ML estimator of $\boldsymbol{\alpha}$ can be expressed as:

$$
\hat{\boldsymbol{\alpha}}=\mathbf{H}_{1} \mathbf{z}+\mathbf{H}_{2} \mathbf{z}^{*},
$$

where $\mathbf{H}_{1} \stackrel{\text { def }}{=} \mathbf{K X}^{H} \mathbf{G}+\mathbf{L} \mathbf{X}^{T} \mathbf{P}^{*}$ and $\mathbf{H}_{2} \stackrel{\text { def }}{=} \mathbf{K} \mathbf{X}^{H} \mathbf{P}+$ $\mathbf{L X}^{T} \mathbf{G}^{*}$.

Since $\hat{\boldsymbol{\alpha}}$ is widely linear of multivariate singular NC-CES distributed vector $\mathbf{z}$, the non-singular ML estimator (48) is singular NC-CES distributed

$$
\hat{\boldsymbol{\alpha}} \sim \mathrm{EC}_{n}^{m-n}\left(\boldsymbol{\alpha}, \mathbf{C}_{\boldsymbol{\alpha}}, \mathbf{C}_{\boldsymbol{\alpha}}^{\prime}, g\right),
$$

where $\mathbf{C}_{\boldsymbol{\alpha}}=\mathbf{H}_{1} \boldsymbol{\Sigma} \mathbf{H}_{1}^{H}+\mathbf{H}_{1} \boldsymbol{\Omega} \mathbf{H}_{2}^{H}+\mathbf{H}_{2} \boldsymbol{\Omega}^{*} \mathbf{H}_{1}^{H}+\mathbf{H}_{2} \boldsymbol{\Sigma}^{*} \mathbf{H}_{2}^{H}$ $\mathbf{C}_{\boldsymbol{\alpha}}^{\prime}=\mathbf{H}_{1} \boldsymbol{\Omega} \mathbf{H}_{1}^{T}+\mathbf{H}_{1} \boldsymbol{\Sigma} \mathbf{H}_{2}^{T}+\mathbf{H}_{2} \boldsymbol{\Sigma}^{*} \mathbf{H}_{1}^{T}+\mathbf{H}_{2} \boldsymbol{\Omega}^{*} \mathbf{H}_{2}^{T}$.

The augmented residuals vector for the model (39) can be defined as

$$
\tilde{\mathbf{e}}=\left(\mathbf{e}^{T} \mathbf{e}^{H}\right)^{T} \stackrel{\text { def }}{=} \tilde{\mathbf{z}}-\tilde{\mathbf{X}} \widehat{\tilde{\boldsymbol{\alpha}}}=\tilde{\mathbf{H}} \tilde{\mathbf{z}},
$$

where $\tilde{\mathbf{H}} \stackrel{\text { def }}{=} \mathbf{I}-\tilde{\mathbf{X}}\left(\tilde{\mathbf{X}}^{H} \tilde{\boldsymbol{\Gamma}}^{\#} \tilde{\mathbf{X}}\right)^{-1} \tilde{\mathbf{X}}^{H} \tilde{\boldsymbol{\Gamma}}^{\#}=\left(\begin{array}{cc}\overline{\mathbf{H}}_{1} & \overline{\mathbf{H}}_{2} \\ \overline{\mathbf{H}}_{2}^{*} & \overline{\mathbf{H}}_{1}^{*}\end{array}\right)$ is idempotent matrix with $\operatorname{rank} \operatorname{rank}(\tilde{\mathbf{H}})=2 \operatorname{rank}\left(\overline{\mathbf{H}}_{1}\right)=2(m-$ $n)$, where $\overline{\mathbf{H}}_{1} \stackrel{\text { def }}{=} \mathbf{I}-\mathbf{X H}_{1}$ and $\overline{\mathbf{H}}_{2} \stackrel{\text { def }}{=} \mathbf{X H}_{2}$. Note that $\tilde{\mathbf{H}} \tilde{\mathbf{X}}=\mathbf{O}$ implies that $\overline{\mathbf{H}}_{1} \mathbf{X}=\mathbf{O}$ and $\overline{\mathbf{H}}_{2} \mathbf{X}^{*}=\mathbf{O}$. By similar steps in the derivation of (48) and (49), the $(m \times 1)$ vector $\mathbf{e}$ of the augmented residuals vector in (50) can be expressed as

$$
\mathbf{e}=\overline{\mathbf{H}}_{1} \mathbf{z}+\overline{\mathbf{H}}_{2} \mathbf{z}^{*},
$$

Consequently, the distribution of $\mathbf{e}$ is given by

$$
\mathbf{e} \sim \mathrm{EC}_{m}^{m-n}\left(\mathbf{0}, \mathbf{C}_{\boldsymbol{e}}, \mathbf{C}_{\boldsymbol{e}}^{\prime}, g\right),
$$

with $\mathbf{C}_{\boldsymbol{e}}=\overline{\mathbf{H}}_{1} \boldsymbol{\Sigma} \overline{\mathbf{H}}_{1}^{H}+\overline{\mathbf{H}}_{1} \boldsymbol{\Omega} \overline{\mathbf{H}}_{2}^{H}+\overline{\mathbf{H}}_{2} \boldsymbol{\Omega}^{*} \overline{\mathbf{H}}_{1}^{H}+\overline{\mathbf{H}}_{2} \boldsymbol{\Sigma}^{*} \overline{\mathbf{H}}_{2}^{H}$ $\mathbf{C}_{\boldsymbol{e}}^{\prime}=\overline{\mathbf{H}}_{1} \boldsymbol{\Omega} \overline{\mathbf{H}}_{1}^{T}+\overline{\mathbf{H}}_{1} \boldsymbol{\Sigma} \overline{\mathbf{H}}_{2}^{T}+\overline{\mathbf{H}}_{2} \boldsymbol{\Sigma}^{*} \overline{\mathbf{H}}_{1}^{T}+\overline{\mathbf{H}}_{2} \boldsymbol{\Omega}^{*} \overline{\mathbf{H}}_{2}^{T}$.

Since $\tilde{\boldsymbol{\Gamma}}=\sigma_{\varepsilon}^{2} \tilde{\boldsymbol{\Gamma}}^{\prime} \stackrel{\text { def }}{=} \sigma_{\varepsilon}^{2}\left(\begin{array}{cc}\boldsymbol{\Sigma}^{\prime} & \boldsymbol{\Omega}^{\prime} \\ \boldsymbol{\Omega}^{\prime *} & \boldsymbol{\Sigma}^{\prime *}\end{array}\right)$, it follows from (50) that

$$
\begin{aligned}
\mathrm{E}\left(\tilde{\mathbf{e}}^{H}\left(\tilde{\mathbf{H}} \tilde{\boldsymbol{\Gamma}}^{\prime} \tilde{\mathbf{H}}^{H}\right)^{\#} \tilde{\mathbf{e}}\right) & =\operatorname{Tr}\left(\left(\tilde{\mathbf{H}} \tilde{\boldsymbol{\Gamma}}^{\prime} \tilde{\mathbf{H}}^{H}\right)^{\#} \mathrm{E}\left(\tilde{\mathbf{e}} \tilde{\mathbf{e}}^{H}\right)\right) \\
& =\sigma_{\varepsilon}^{2} c_{0} \operatorname{Tr}\left(\left(\tilde{\mathbf{H}} \tilde{\boldsymbol{\Gamma}}^{\prime} \tilde{\mathbf{H}}^{H}\right)^{\#}\left(\tilde{\mathbf{H}} \tilde{\boldsymbol{\Gamma}}^{\prime} \tilde{\mathbf{H}}^{H}\right)\right) \\
& =2 \sigma_{\varepsilon}^{2} c_{0}(m-n)
\end{aligned}
$$

where $c_{0}$ is a real positive constant defined in (47) which takes different values according to the choices of CES distributions. Therefore the following statistic $\hat{\sigma}_{\varepsilon}^{2}$ defined in (51) is an unbiased estimator of $\sigma_{\varepsilon}^{2}$

$$
\hat{\sigma}_{\varepsilon}^{2}=\frac{\tilde{\mathbf{e}}^{H}\left(\tilde{\mathbf{H}}^{H} \tilde{\boldsymbol{\Gamma}}^{\prime} \tilde{\mathbf{H}}\right)^{\#} \tilde{\mathbf{e}}}{2 c_{0}(m-n)} .
$$

Since the NC-CCG distributions presented in section 4 form a subclass of the CES distributions, it follows from result 9 that, if $\varepsilon \sim \mathbb{C N}_{m}^{r}(\mathbf{0}, \boldsymbol{\Sigma}, \boldsymbol{\Omega})$, the quadratic from $\frac{\tilde{\mathbf{e}}^{H}\left(\tilde{\mathbf{H}}_{\mathbf{\Gamma}} \tilde{\mathbf{\Gamma}}^{\prime} \tilde{\mathbf{H}}^{H}\right)^{\#} \tilde{\mathbf{e}}}{\sigma_{\varepsilon}^{2}}$ has the following representation

$$
\frac{\tilde{\mathbf{e}}^{H}\left(\tilde{\mathbf{H}} \tilde{\boldsymbol{\Gamma}}^{\prime} \tilde{\mathbf{H}}^{H}\right)^{\#} \tilde{\mathbf{e}}}{\sigma_{\varepsilon}^{2}}={ }_{d} \tau \chi_{q_{c}}^{2},
$$

where $q_{c}=\operatorname{rank}(\tilde{\mathbf{H}})=2(m-n)$. Therefore, the statistic $\hat{\sigma}_{\varepsilon}^{2}$ in (45) remains unbiased estimator of $\sigma_{\varepsilon}^{2}$ where $c_{0}=\mathrm{E}(\tau)$.

Fig.1. illustrates the estimated of binary phase-shift keying (BPSK) and quadrature Phase shift keying (QPSK) signals, $\alpha$, using (48) for the underlying complex-valued linear model (38) with error term $\varepsilon$ following one of the three distributions: singular NC-CN distribution $\left(\mathbb{C N}_{6}^{3}(\mathbf{0}, \boldsymbol{\Sigma}, \boldsymbol{\Omega})\right.$ ), singular noncircular complex t-distribution $\left(\mathbb{C} t_{6,5}^{3}(\mathbf{0}, \boldsymbol{\Sigma}, \boldsymbol{\Omega})\right.$ ) (singular circular complex t-distribution is obtained when $\Omega=\mathbf{O}$ ), parameter vector $\boldsymbol{\alpha}$ consists of 2 identically independently distributed BPSK symbols, each out $\{+1,-1\}$ or QPSK symbols, each out of $\{ \pm 1 \pm j\}$ and a $6 \times 2$ know matrix $\mathbf{X}$ of full column rank. The matrices $\boldsymbol{\Sigma}$ and $\boldsymbol{\Omega}$ are defined as $\boldsymbol{\Sigma}=\sigma_{\varepsilon}^{2} \mathbf{A} \mathbf{A}^{H}$ and $\boldsymbol{\Omega}=\sigma_{\varepsilon}^{2} \mathbf{A} \boldsymbol{\Delta}_{3} \mathbf{A}^{T}$ with $\mathbf{A} \stackrel{\text { def }}{=}\left(\mathbf{a}_{1}, \mathbf{a}_{2}, \mathbf{a}_{3}\right)$ and $\boldsymbol{\Delta}_{3} \stackrel{\text { def }}{=}$ $\operatorname{Diag}(0.7,0.6,0.9)$ where $a_{k} \stackrel{\text { def }}{=}\left(1, e^{j \theta_{k}}, \ldots, e^{j(M-1) \theta_{k}}\right)^{T}$. The two last distributions are normalized so that $\operatorname{Cov}(\varepsilon)=\Sigma$ and $\operatorname{pcov}(\varepsilon)=\Omega$ (i.e., $c_{0}=1$ ). It can be seen from Fig. 1 that the estimates $\hat{\boldsymbol{\alpha}}$ are centered around the true constellation points in the presence of the three distributed error terms. Fig. 2 compares the minimum square error (MSE) $\mathrm{E}\left((\hat{\boldsymbol{\alpha}}-\boldsymbol{\alpha})^{H}(\hat{\boldsymbol{\alpha}}-\boldsymbol{\alpha})\right)$ associated with the circular ML estimate (41) and non-circular ML estimate (48) of $\boldsymbol{\alpha}$ and the theoretical circular and non-circular bounds given respectively by (42) and (47). As can be seen in this figures, the MSE reaches the theoretical circular bound [resp. non-circular bound] for the three singular C-CES [resp. singular NC-CES] distributed error terms.

\section{Conclusion}

Absolutely continuous singular NC-CES distributions are presented by deriving explicit expressions for its p.d.f's. The 

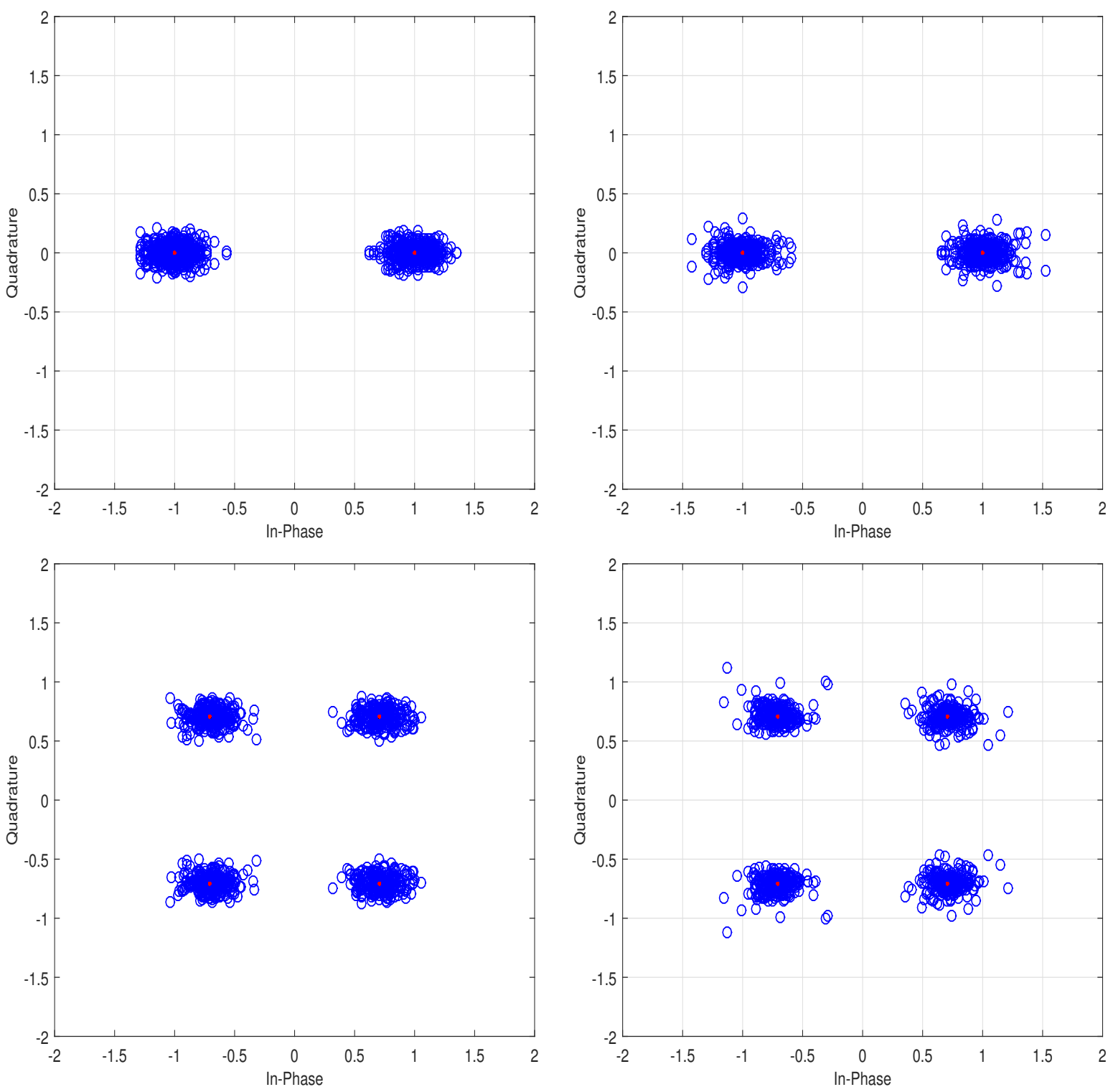

(a)

(b)

Figure 1. The ML estimates of BPSK (first row) and QPSK (second row) signals that are obtained using (48), in the presence of the three error terms: $\mathbb{C} \mathcal{N}_{6}^{3}(\mathbf{0}, \boldsymbol{\Sigma}, \boldsymbol{\Omega})\left(\right.$ column (a)), and $\mathbb{C} t_{6,5}^{3}(\mathbf{0}, \boldsymbol{\Sigma}, \boldsymbol{\Omega})(\operatorname{column}(\mathrm{b}))$ with $\sigma_{\varepsilon}^{2}=0.01$.

stochastic representation of the singular NC-CES distributions and quadratic forms in NC-CES r.v. are proved. As special cases, explicit expressions for the p.d.f's of multivariate complex r.v.'s with singular NC-CN distribution and singular NCCCG distribution are also presented. Some useful properties of singular NC-CES distributions and their conditional distributions are also derived. The singular C-CES distributions are presented as special cases of NC-CES distributions. Singular widely linear mean square estimators of a signal from singular non-circular or circular distributed measurement data vector are derived. The problem of estimating the parameters of a complex-valued non-circular multivariate linear model in the presence either of singular NC-CES or C-CES distributed error terms is presented and followed by deriving widely linear estimators.

\section{A Proof of Lemma 1}

Since $\boldsymbol{\Sigma}$ is singular with $\operatorname{rank}(\boldsymbol{\Sigma})=r$, the matrix $\boldsymbol{\Sigma}$ can be decomposed via eigenvalue decomposition as

$$
\begin{aligned}
\boldsymbol{\Sigma} & =\left(\begin{array}{ll}
\mathbf{U}_{r} & \overline{\mathbf{U}}_{r}
\end{array}\right)\left(\begin{array}{cc}
\boldsymbol{\Lambda}_{r} & \mathbf{O} \\
\mathbf{O} & \mathbf{O}
\end{array}\right)\left(\begin{array}{c}
\mathbf{U}_{r}^{H} \\
\overline{\mathbf{U}}_{r}^{H}
\end{array}\right) \\
& =\mathbf{U}_{r} \boldsymbol{\Lambda}_{r} \mathbf{U}_{r}^{H},
\end{aligned}
$$

where the columns of the complex matrix $\overline{\mathbf{U}}_{r} \in \mathbb{C}^{m \times m-r}$ are the eigenvectors corresponding to the zero eigenvalues, therefore $\boldsymbol{\Sigma} \overline{\mathbf{U}}_{r}=\mathbf{O}$. Let $\mathbf{C}$ be an $(m \times m)$ matrix defined as

$$
\mathbf{C} \stackrel{\text { def }}{=} \boldsymbol{\Sigma}^{\# \frac{1}{2}} \boldsymbol{\Omega} \boldsymbol{\Sigma}^{\# \frac{T}{2}}
$$

where $\boldsymbol{\Sigma}^{\# \frac{1}{2}}=\mathbf{U}_{r} \boldsymbol{\Lambda}_{r}^{-\frac{1}{2}} \mathbf{U}_{r}^{H}$. Since $\mathbf{C}$ is a complex symmetric matrix of rank $p$, by Takagi factorization [35] there exists a 


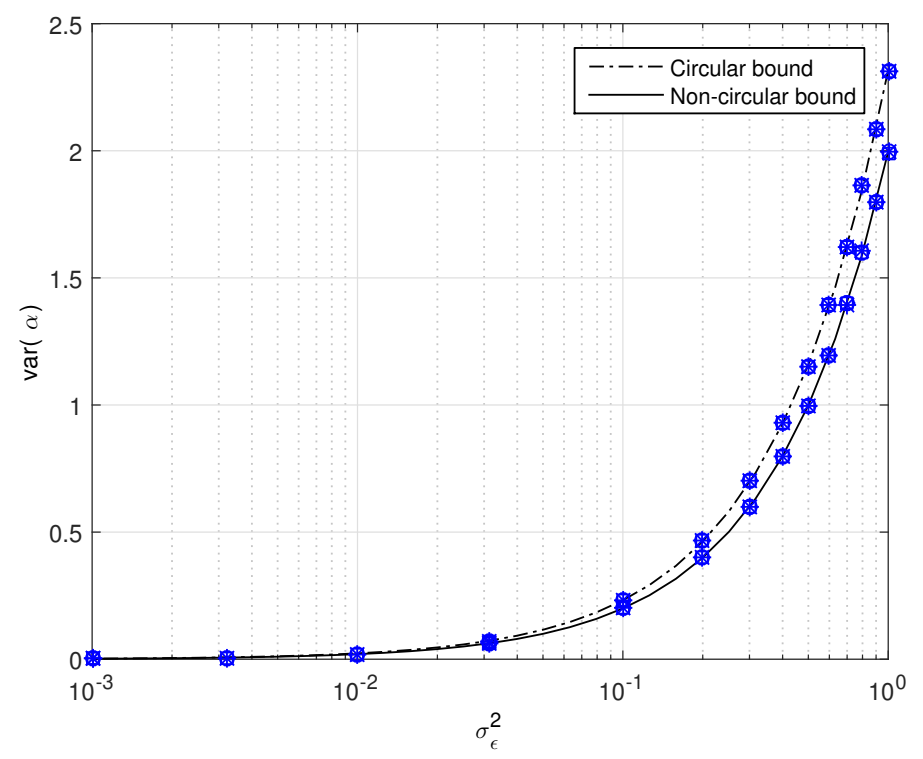

Figure 2. The MSE of the ML estimate QPSK signal vector $\boldsymbol{\alpha}$, the theoretical circular bound (42) and non-circular bound (47) in the presence of the three error terms: $\mathbb{C N}_{6}^{3}(\mathbf{0}, \boldsymbol{\Sigma}, \boldsymbol{\Omega})(*), \mathbb{C} t_{6, \nu}^{3}(\mathbf{0}, \boldsymbol{\Sigma}, \boldsymbol{\Omega})(\square)$ and $\mathbb{C} K_{6, \nu}^{3}(\mathbf{0}, \boldsymbol{\Sigma}, \boldsymbol{\Omega})(\circ)$ versus $\sigma_{\varepsilon}^{2}$ where the number of Monte-Carlo iterations is fixed at 100

unitary matrix $\mathbf{S}=\left(\begin{array}{cc}\mathbf{S}_{p} & \overline{\mathbf{S}}_{p}\end{array}\right)$ and a $\boldsymbol{\Delta}=\left(\begin{array}{cc}\boldsymbol{\Delta}_{p} & \mathbf{O} \\ \mathbf{O} & \mathbf{O}\end{array}\right)$ nonnegative diagonal matrix such that

$$
\begin{aligned}
\mathbf{C} & =\mathbf{S} \boldsymbol{\Delta} \mathbf{S}^{T}=\left(\begin{array}{ll}
\mathbf{S}_{p} & \overline{\mathbf{S}}_{p}
\end{array}\right)\left(\begin{array}{cc}
\boldsymbol{\Delta}_{p} & \mathbf{O} \\
\mathbf{O} & \mathbf{O}
\end{array}\right)\left(\begin{array}{c}
\mathbf{S}_{p}^{T} \\
\overline{\mathbf{S}}_{p}^{T}
\end{array}\right) \\
& =\mathbf{S}_{p} \boldsymbol{\Delta}_{p} \overline{\mathbf{S}}_{p}^{T}
\end{aligned}
$$

where $\boldsymbol{\Delta}_{p} \stackrel{\text { def }}{=} \operatorname{Diag}\left(\kappa_{1}, \ldots, \kappa_{p}\right)$ with $\kappa_{l} \neq 0$, and $\left|\kappa_{l}\right|<1$ for $l=1, \ldots, p$ and $\mathbf{S}_{p} \in \mathbb{C}^{m \times p}$ and $\overline{\mathbf{S}}_{p} \in \mathbb{C}^{m \times(m-p)}$ are matrices with orthonormal columns.

Thus,

$$
\mathbf{U}_{r} \boldsymbol{\Lambda}_{r}^{-\frac{1}{2}} \mathbf{U}_{r}^{H} \boldsymbol{\Omega} \mathbf{U}_{r}^{*} \boldsymbol{\Lambda}_{r}^{-\frac{1}{2}} \mathbf{U}_{r}^{T}=\mathbf{S}_{p} \boldsymbol{\Delta}_{p} \mathbf{S}_{p}^{T} .
$$

Since $\operatorname{Span}\left(\mathbf{S}_{p}\right) \subseteq \operatorname{Span}\left(\mathbf{U}_{r}\right)$, there exists a matrix $\mathbf{V}_{p} \in$ $\mathbb{C}^{r \times p}$ with orthonormal columns such that $\mathbf{S}_{p}=\mathbf{U}_{r} \mathbf{V}_{p}$. Hence, (53) becomes

$$
\mathbf{U}_{r}^{H} \boldsymbol{\Omega} \mathbf{U}_{r}^{*}=\Lambda_{r}^{\frac{1}{2}} \mathbf{V}_{p} \boldsymbol{\Delta}_{p} \mathbf{V}_{p}^{T} \boldsymbol{\Lambda}_{r}^{\frac{1}{2}}
$$

Therefore, $\Omega$ can be expressed as

$$
\boldsymbol{\Omega}=\mathbf{U}_{r} \boldsymbol{\Lambda}_{r}^{\frac{1}{2}} \mathbf{V}_{p} \boldsymbol{\Delta}_{p} \mathbf{V}_{p}^{T} \boldsymbol{\Lambda}_{r}^{\frac{1}{2}} \mathbf{U}_{r}^{T}
$$

\section{B Proof of result 2}

Since $\boldsymbol{\Sigma}$ is singular with $\operatorname{rank}(\boldsymbol{\Sigma})=r, \boldsymbol{\Sigma}$ can be decomposed into the product of matrices $\mathbf{U} \stackrel{\text { def }}{=}\left(\begin{array}{lll}\mathbf{U}_{r} & \overline{\mathbf{U}}_{r}\end{array}\right)$ and $\boldsymbol{\Lambda} \stackrel{\text { def }}{=}\left(\begin{array}{cc}\boldsymbol{\Lambda}_{r} & \mathbf{O} \\ \mathbf{O} & \mathbf{O}\end{array}\right)$ as shown in (52).

Recall that an affine linear transformation of NC-CES distribution is NC-CES distribution too [11, Theorem 1] (i.e., $\mathbf{z} \sim \mathrm{EC}_{m}(\boldsymbol{\mu}, \boldsymbol{\Sigma}, \boldsymbol{\Omega}, g)$ then $\mathbf{B z}+\mathbf{b} \sim \mathrm{EC}_{m}(\mathbf{B} \boldsymbol{\mu}+$ $\left.\mathbf{b}, \mathbf{B} \boldsymbol{\Sigma} \mathbf{B}^{H}, \mathbf{B} \boldsymbol{\Omega} \mathbf{B}^{T}, g\right)$ for all $\mathbf{B} \in \mathbb{C}^{N \times m}$ and $\mathbf{b} \in \mathbb{C}^{m}$ and non-singular $\left.\mathbf{B} \in \mathbb{C}^{m \times m}\right)$. Therefore, $\overline{\mathbf{z}} \stackrel{\text { def }}{=}\left(\mathbf{z}_{1}^{H} \mathbf{z}_{2}^{H}\right)^{H}=$
$\mathbf{U}^{H} \mathbf{z}=\left(\mathbf{z}^{H} \mathbf{U}_{r} \quad \mathbf{z}^{H} \overline{\mathbf{U}}_{r}\right)^{H} \sim \mathrm{EC}_{m}\left(\mathbf{U}^{H} \boldsymbol{\mu}, \boldsymbol{\Sigma}_{u}, \boldsymbol{\Omega}_{u}, g\right)$ where $\boldsymbol{\Sigma}_{u} \stackrel{\text { def }}{=} \mathbf{U}^{H} \boldsymbol{\Sigma} \mathbf{U}$ and $\boldsymbol{\Omega}_{u} \stackrel{\text { def }}{=} \mathbf{U}^{H} \boldsymbol{\Omega} \mathbf{U}^{*}$ are hermitian and complex symmetric matrices, respectively. Using the structure of the matrix $\mathbf{U}$ and (52), $\boldsymbol{\Sigma}_{u}$ and $\boldsymbol{\Omega}_{u}$ can be partitioned as

$$
\begin{aligned}
\boldsymbol{\Sigma}_{u} & =\left(\begin{array}{cc}
\boldsymbol{\Lambda}_{r} & \mathbf{O} \\
\mathbf{O} & \mathbf{O}
\end{array}\right) \\
\boldsymbol{\Omega}_{u} & =\left(\begin{array}{c}
\mathbf{U}_{r}^{H} \\
\overline{\mathbf{U}}_{r}^{H}
\end{array}\right) \boldsymbol{\Omega}\left(\begin{array}{ll}
\mathbf{U}_{r}^{*} & \overline{\mathbf{U}}_{r}^{*}
\end{array}\right) \\
& =\left(\begin{array}{cc}
\mathbf{U}_{r}^{H} \boldsymbol{\Omega} \mathbf{U}_{r}^{*} & \mathbf{U}_{r}^{H} \boldsymbol{\Omega} \overline{\mathbf{U}}_{r}^{*} \\
\overline{\mathbf{U}}_{r}^{H} \boldsymbol{\Omega} \mathbf{U}_{r}^{*} & \overline{\mathbf{U}}_{r}^{H} \boldsymbol{\Omega} \overline{\mathbf{U}}_{r}^{*}
\end{array}\right) \\
& =\left(\begin{array}{cc}
\mathbf{U}_{r}^{H} \boldsymbol{\Omega} \mathbf{U}_{r}^{*} & \mathbf{O} \\
\mathbf{O} & \mathbf{O}
\end{array}\right)
\end{aligned}
$$

using $\mathbf{U}_{r}^{H} \boldsymbol{\Omega} \overline{\mathbf{U}}_{r}^{*}=\mathbf{O}$ and $\overline{\mathbf{U}}_{r}^{H} \boldsymbol{\Omega} \overline{\mathbf{U}}_{r}^{*}=\mathbf{O}$, thanks to lemma 1 and $\overline{\mathbf{U}}_{r}^{H} \mathbf{U}_{r}=\mathbf{O}$. Applying theorem [11, Theorem 2], yields $\mathbf{z}_{2} \sim \mathrm{EC}_{m}^{r}\left(\overline{\mathbf{U}}^{H} \boldsymbol{\mu}, \mathbf{O}, \mathbf{O}, g\right)$. In other words,

$$
\mathbf{z}_{2}-\overline{\mathbf{U}}^{H} \boldsymbol{\mu}=\overline{\mathbf{U}}^{H}(\mathbf{z}-\boldsymbol{\mu})=\mathbf{0} \text { w.p.1 }
$$

or, equivalently, $\mathbf{z}-\boldsymbol{\mu} \in\left(\operatorname{Im} \overline{\mathbf{U}}_{r}\right)^{\perp}=\operatorname{Im} \boldsymbol{\Sigma}$. Thus the p.d.f. of $\mathbf{z}_{2}$ is given by (54). Furthermore, once again by theorem [11, Theorem 2], $\mathbf{z}_{1} \sim \mathrm{EC}_{m}^{r}\left(\mathbf{U}_{r}{ }^{H} \boldsymbol{\mu}, \boldsymbol{\Lambda}_{r}, \mathbf{U}_{r}^{H} \boldsymbol{\Omega} \mathbf{U}_{r}^{*}, g\right)$. Since the digonal matrice $\boldsymbol{\Lambda}_{r}$ and its chur complement matrix $\mathbf{P}_{r} \stackrel{\text { def }}{=}$ $\boldsymbol{\Lambda}_{r}-\left(\mathbf{U}_{r}^{T} \boldsymbol{\Omega}^{H} \mathbf{U}_{r}\right) \boldsymbol{\Lambda}_{r}^{-1}\left(\mathbf{U}_{r}^{H} \boldsymbol{\Omega} \mathbf{U}_{r}^{*}\right)$ are non-singular, the matrix

$$
\boldsymbol{\Gamma}_{r} \stackrel{\text { def }}{=}\left(\begin{array}{cc}
\boldsymbol{\Lambda}_{r} & \mathbf{U}_{r}^{H} \boldsymbol{\Omega U}_{r}^{*} \\
\mathbf{U}_{r}^{T} \boldsymbol{\Omega}^{*} \mathbf{U}_{r} & \boldsymbol{\Lambda}_{r}
\end{array}\right)
$$

is non-singular and consequently the p.d.f. of $\mathbf{z}_{1}$ exists and by using (2),

$$
\left.p\left(\mathbf{z}_{1}\right)=c_{r, g} \operatorname{det}\left(\boldsymbol{\Gamma}_{r}^{-\frac{1}{2}}\right) g\left(q\left(\mathbf{z}_{1}\right)\right)\right)
$$

where $q\left(\mathbf{z}_{1}\right)$ is a quadratic form $q\left(\mathbf{z}_{1}\right) \stackrel{\text { def }}{=} \frac{1}{2}\left(\tilde{\mathbf{z}}_{1}-\right.$ $\left.\tilde{\mathbf{U}}_{r}^{H} \tilde{\boldsymbol{\mu}}\right)^{H} \boldsymbol{\Gamma}_{r}^{-1}\left(\tilde{\mathbf{z}}_{1}-\tilde{\mathbf{U}}_{r}^{H} \tilde{\boldsymbol{\mu}}\right), \quad \tilde{\mathbf{z}}_{1}=\tilde{\mathbf{U}}_{r}^{H} \tilde{\mathbf{z}}$ and $\tilde{\mathbf{U}}_{r}=$ 


$$
\begin{aligned}
& \left(\begin{array}{cc}
\mathbf{U}_{r} & \mathbf{O} \\
\mathbf{O} & \mathbf{U}_{r}^{*}
\end{array}\right) . \text { Note that } \\
& \qquad \begin{aligned}
q\left(\mathbf{z}_{1}\right) & =\frac{1}{2}\left(\tilde{\mathbf{z}}_{1}-\tilde{\mathbf{U}}_{r}^{H} \tilde{\boldsymbol{\mu}}\right)^{H} \boldsymbol{\Gamma}_{r}^{-1}\left(\tilde{\mathbf{z}}_{1}-\tilde{\mathbf{U}}_{r}^{H} \tilde{\boldsymbol{\mu}}\right) \\
& =\frac{1}{2}(\tilde{\mathbf{z}}-\tilde{\boldsymbol{\mu}})^{H} \tilde{\mathbf{U}}_{r} \boldsymbol{\Gamma}_{r}^{-1} \tilde{\mathbf{U}}_{r}^{H}(\tilde{\mathbf{z}}-\tilde{\boldsymbol{\mu}}) .
\end{aligned}
\end{aligned}
$$

Using the fact that

$$
\boldsymbol{\Gamma}^{\#}=\tilde{\mathbf{U}}_{r} \boldsymbol{\Gamma}_{r}^{-1} \tilde{\mathbf{U}}_{r}^{H}=\tilde{\mathbf{U}}_{r}\left(\tilde{\mathbf{U}}_{r}^{H} \tilde{\boldsymbol{\Gamma}}_{r}\right)^{-1} \tilde{\mathbf{U}}_{r}^{H},
$$

$q\left(\mathbf{z}_{1}\right)$ becomes

$$
q\left(\mathbf{z}_{1}\right)=\frac{1}{2}(\tilde{\mathbf{z}}-\tilde{\boldsymbol{\mu}})^{H} \boldsymbol{\Gamma}^{\#}(\tilde{\mathbf{z}}-\tilde{\boldsymbol{\mu}})=q_{s}^{n c}(\mathbf{z}) .
$$

It follows from lemma 1 that $\mathbf{P}_{r}$ can be expressed after replacing $\Omega$ by its expression as

$$
\begin{aligned}
\mathbf{P}_{r} & =\boldsymbol{\Lambda}_{r}-\left(\mathbf{U}_{r}^{T} \boldsymbol{\Omega}^{H} \mathbf{U}_{r}\right) \boldsymbol{\Lambda}_{r}^{-1}\left(\mathbf{U}_{r}^{H} \boldsymbol{\Omega} \mathbf{U}_{r}^{*}\right) \\
& =\boldsymbol{\Lambda}_{r}^{\frac{1}{2}} \mathbf{V}_{p}^{*}\left(\mathbf{I}-\overline{\boldsymbol{\Delta}}_{p}\right) \mathbf{V}_{p}^{T} \boldsymbol{\Lambda}_{r}^{\frac{1}{2}},
\end{aligned}
$$

where $\overline{\boldsymbol{\Delta}}_{p} \stackrel{\text { def }}{=} \operatorname{Diag}\left(\left|\kappa_{1}\right|^{2}, \ldots,\left|\kappa_{p}\right|^{2}\right)$. Using (59), it follows from the result for the determinant of a partitioned matrix [40] that

$$
\begin{aligned}
\operatorname{det}\left(\boldsymbol{\Gamma}_{r}\right) & =\operatorname{det}\left(\boldsymbol{\Gamma}_{r}\right) \operatorname{det}\left(\mathbf{P}_{r}\right)=\left(\operatorname{det}\left(\boldsymbol{\Gamma}_{r}\right)\right)^{2} \operatorname{det}\left(\mathbf{I}-\overline{\mathbf{\Delta}}_{p}\right) \\
& =\prod_{k=1}^{r} \lambda_{k}^{2} \prod_{l=1}^{p}\left(1-\left|\kappa_{l}\right|^{2}\right) .
\end{aligned}
$$

By substituting (58) and (60) into (56) yields the p.d.f. (8) for singular NC-CES distributions defined on a subspace (54).

\section{Proof of Corollary 1}

Let us prove the first point. Using (3), $\mathbf{v}^{(r)}$ in (11) can be expressed as

$$
\mathbf{v}^{(r)}=\boldsymbol{\Delta}_{1}^{(r)} \mathbf{u}^{(r)}+\boldsymbol{\Delta}_{2}^{(r)}\left(\mathbf{u}^{(r)}\right)^{*},
$$

where $\boldsymbol{\Delta}_{1}^{(r)}=\frac{\boldsymbol{\Delta}_{+}^{(r)}+\boldsymbol{\Delta}_{-}^{(r)}}{2}, \boldsymbol{\Delta}_{2}^{(r)}=\frac{\boldsymbol{\Delta}_{+}^{(r)}-\boldsymbol{\Delta}_{-}^{(r)}}{2}$ and where $\boldsymbol{\Delta}_{+}^{(r)} \stackrel{\text { def }}{=} \sqrt{\mathbf{I}+\Delta_{r}}, \boldsymbol{\Delta}_{+}^{(r)} \stackrel{\text { def }}{=} \sqrt{\mathbf{I}-\Delta_{r}}$. It follows from (11) that the extended vector $\tilde{\mathbf{z}}$ admits the following stochastic representation

$$
\tilde{\mathbf{z}}=\tilde{\boldsymbol{\mu}}+\mathcal{R} \tilde{\mathbf{U}}_{r} \tilde{\mathbf{W}}_{r} \tilde{\boldsymbol{\Delta}}_{r} \tilde{\mathbf{u}}^{(r)},
$$

where $\quad \tilde{\mathbf{W}}_{r} \quad \stackrel{\text { def }}{=}\left(\begin{array}{cc}\boldsymbol{\Lambda}_{r}^{\frac{1}{2}} \mathbf{V}_{r} & \mathbf{O} \\ \mathbf{O} & \boldsymbol{\Lambda}_{r}^{\frac{1}{2}} \mathbf{V}_{r}^{*}\end{array}\right), \quad \tilde{\boldsymbol{\Delta}}_{r} \stackrel{\text { def }}{=}$ $\left(\begin{array}{ll}\boldsymbol{\Delta}_{1}^{(r)} & \boldsymbol{\Delta}_{2}^{(r)} \\ \boldsymbol{\Delta}_{2}^{(r)} & \boldsymbol{\Delta}_{1}^{(r)}\end{array}\right)$ and $\tilde{\mathbf{u}}^{(r)} \stackrel{\text { def }}{=}\left(\begin{array}{c}\mathbf{u}^{(r)} \\ \left(\mathbf{u}^{(r)}\right)^{*}\end{array}\right)$.

Substituting (10) into (55), $\boldsymbol{\Gamma}_{r}$ becomes

$$
\boldsymbol{\Gamma}_{r}=\tilde{\mathbf{W}}_{r} \mathbf{C}_{r} \tilde{\mathbf{W}}_{r}^{H},
$$

where $\mathbf{C}_{r} \stackrel{\text { def }}{=}\left(\begin{array}{cc}\mathbf{I} & \boldsymbol{\Delta}_{r} \\ \boldsymbol{\Delta}_{r} & \mathbf{I}\end{array}\right)$. Hence, the Moore-Penrose inverse of $\boldsymbol{\Gamma}$ in (57) can be expressed as

$$
\tilde{\boldsymbol{\Gamma}}^{\#}=\tilde{\mathbf{U}}_{r} \boldsymbol{\Gamma}_{r}^{-1} \tilde{\mathbf{U}}_{r}^{H}=\tilde{\mathbf{U}}_{r}\left(\tilde{\mathbf{W}}_{r}^{H}\right)^{-1} \mathbf{C}_{r}^{-1} \tilde{\mathbf{W}}_{r}^{-1} \tilde{\mathbf{U}}_{r}^{H} .
$$

Then, it follows form (63) and (62) that

$$
(\tilde{\mathbf{z}}-\tilde{\boldsymbol{\mu}})^{H} \tilde{\boldsymbol{\Gamma}}^{\#}(\tilde{\mathbf{z}}-\tilde{\boldsymbol{\mu}})=\mathcal{Q} \tilde{\mathbf{u}}^{H} \tilde{\boldsymbol{\Delta}}_{r}^{T} \mathbf{C}_{r}^{-1} \tilde{\boldsymbol{\Delta}}_{r} \tilde{\mathbf{u}},
$$

using $\tilde{\mathbf{W}}_{r}^{-1} \tilde{\mathbf{W}}_{r}=\mathbf{I}$ and $\tilde{\mathbf{U}}_{r}^{H} \tilde{\mathbf{U}}_{r}=\mathbf{I}$. Simple algebraic manipulation yields

$$
\mathbf{C}_{r}=\tilde{\boldsymbol{\Delta}}_{r}^{T} \tilde{\boldsymbol{\Delta}}_{r},
$$

using the following identities

$$
\begin{aligned}
& \boldsymbol{\Delta}_{1}^{(r)} \boldsymbol{\Delta}_{1}^{(r)}+\boldsymbol{\Delta}_{2}^{(r)} \boldsymbol{\Delta}_{2}^{(r)}=\mathbf{I} \\
& \boldsymbol{\Delta}_{1}^{(r)} \boldsymbol{\Delta}_{2}^{(r)}+\boldsymbol{\Delta}_{2}^{(r)} \boldsymbol{\Delta}_{1}^{(r)}=\boldsymbol{\Delta}_{r} .
\end{aligned}
$$

Therefore, (64) can be simplified as

$$
(\tilde{\mathbf{z}}-\tilde{\boldsymbol{\mu}})^{H} \tilde{\boldsymbol{\Gamma}}^{\#}(\tilde{\mathbf{z}}-\tilde{\boldsymbol{\mu}})=\mathcal{Q} \tilde{\mathbf{u}}^{H} \tilde{\mathbf{u}}={ }_{d} 2 \mathcal{Q},
$$

using $\tilde{\mathbf{u}}^{H} \tilde{\mathbf{u}}=2$. Thus (14) is proved. The second point follows immediately from the first one (14) by taking $\Omega=\mathbf{O}$.

\section{Proof of result 5}

Proof of the first point: Let $\mathbf{Z}_{1}=(\mathbf{I} \mathbf{O})$ and $\mathbf{Z}_{2}=(\mathbf{O} \mathbf{I})$ be two $(m \times d)$ matrices. Then $\mathbf{Z}_{1} \boldsymbol{\mu}=\boldsymbol{\mu}_{1}$ and $\mathbf{Z}_{2} \boldsymbol{\mu}=\boldsymbol{\mu}_{2}$. Since $\operatorname{rank}\left(\boldsymbol{\Sigma}_{11}\right)=r_{1}$ and $\operatorname{rank}\left(\boldsymbol{\Sigma}_{22}\right)=r_{2}$, it follows from result 1 and [11, Theorem 1] that $\mathbf{z}_{1}=\mathbf{Z}_{1} \mathbf{z} \sim \mathrm{EC}_{d}^{r_{1}}\left(\boldsymbol{\mu}_{1}, \boldsymbol{\Sigma}_{11}, \boldsymbol{\Omega}_{11}, g\right)$ and $\mathbf{z}_{2}=\mathbf{Z}_{2} \mathbf{z} \sim \mathrm{EC}_{n}^{r_{2}}\left(\boldsymbol{\mu}_{2}, \boldsymbol{\Sigma}_{22}, \boldsymbol{\Omega}_{22}, g\right)$.

Proof of the second point: Let $\boldsymbol{\Lambda}_{r_{1}}$ and $\boldsymbol{\Lambda}_{r_{2}}$ be two diagonal matrices containing, respectively, the $r_{1}$ and $r_{2}$ nonzero eigenvalues of the matrices $\boldsymbol{\Sigma}_{11}$ and $\boldsymbol{\Sigma}_{22}$, the columns of the two complex matrices $\mathbf{U}_{r, 1} \in \mathbb{C}^{d \times r_{1}}$ and $\mathbf{U}_{r, 2} \in \mathbb{C}^{n \times r_{2}}$ are respectively the corresponding eigenvectors of the $r_{1}$ and $r_{2}$ nonzero eigenvalues of the matrices $\boldsymbol{\Sigma}_{11}$ and $\boldsymbol{\Sigma}_{22}$. Therefore $\boldsymbol{\Sigma}_{11}$ and $\boldsymbol{\Sigma}_{22}$ can be written as $\boldsymbol{\Sigma}_{11}=\mathbf{U}_{r, 1} \boldsymbol{\Lambda}_{r_{1}} \mathbf{U}_{r, 1}^{H}$ and $\boldsymbol{\Sigma}_{22}=\mathbf{U}_{r, 2} \boldsymbol{\Lambda}_{r_{2}} \mathbf{U}_{r, 2}^{H}$. Define $\overline{\mathbf{z}}_{1} \stackrel{\text { def }}{=} \mathbf{U}_{r, 1}^{H} \mathbf{z}_{1}$ and $\overline{\mathbf{z}}_{2} \stackrel{\text { def }}{=} \mathbf{U}_{r, 2}^{H} \mathbf{z}_{2}$. It follows from the first point that $\overline{\mathbf{z}}_{1} \sim$ $\mathrm{EC}_{d}\left(\boldsymbol{\mu}_{1}, \boldsymbol{\Lambda}_{r_{1}}, \overline{\boldsymbol{\Omega}}_{11}, g\right)$ and $\overline{\mathbf{z}}_{2} \sim \mathrm{EC}_{n}\left(\overline{\boldsymbol{\mu}}_{2}, \boldsymbol{\Lambda}_{r_{2}}, \overline{\boldsymbol{\Omega}}_{22}, g\right)$ where $\overline{\boldsymbol{\mu}}_{1} \stackrel{\text { def }}{=} \mathbf{U}_{r, 1}^{H} \boldsymbol{\mu}_{1}, \overline{\boldsymbol{\mu}}_{2} \stackrel{\text { def }}{=} \mathbf{U}_{r, 2}^{H} \boldsymbol{\mu}_{2}, \overline{\boldsymbol{\Omega}}_{11} \stackrel{\text { def }}{=} \mathbf{U}_{r, 1}^{H} \boldsymbol{\Omega}_{11} \mathbf{U}_{r, 1}^{*}$ and $\overline{\boldsymbol{\Omega}}_{22} \stackrel{\text { def }}{=} \mathbf{U}_{r, 2}^{H} \boldsymbol{\Omega}_{22} \mathbf{U}_{r, 2}^{*}$. Since $\overline{\mathbf{z}}_{1}$ and $\overline{\mathbf{z}}_{2}$ have non-singular NC-CES distributions, it follows from [11] that $\overline{\mathbf{z}}_{1} \mid \overline{\mathbf{z}}_{2} \sim$ $\mathrm{EC}_{d}\left(\boldsymbol{\mu}_{\bar{z}_{1} \mid \bar{z}_{2}}, \overline{\boldsymbol{\Sigma}}_{11.2}, \overline{\boldsymbol{\Omega}}_{11.2}, g_{1 \mid 2}\right)$ where $\boldsymbol{\mu}_{\bar{z}_{1} \mid \bar{z}_{2}}, \overline{\boldsymbol{\Sigma}}_{11.2}$ and $\overline{\boldsymbol{\Omega}}_{11.2}$ will be determined appropriately below. It also follows from $[30$, rels. (2.83)-(2.84)] that the augmented conditional vector is given by

$$
\tilde{\overline{\boldsymbol{\mu}}}_{1 \mid 2}=\tilde{\overline{\boldsymbol{\mu}}}_{1}+\tilde{\boldsymbol{\Gamma}}_{\bar{z}_{1} \bar{z}_{2}} \tilde{\boldsymbol{\Gamma}}_{\bar{z}_{2}}^{-1}\left(\tilde{\overline{\mathbf{z}}}_{2}-\tilde{\overline{\boldsymbol{\mu}}}_{\mathbf{2}}\right),
$$

and the conditional augmented scattered matrix is given by

$$
\tilde{\boldsymbol{\Gamma}}_{\bar{z}_{1} \bar{z}_{1} \mid \bar{z}_{2}}=\tilde{\boldsymbol{\Gamma}}_{\bar{z}_{1}}-\tilde{\boldsymbol{\Gamma}}_{\bar{z}_{1} \bar{z}_{2}} \tilde{\boldsymbol{\Gamma}}_{\bar{z}_{2}}^{-1} \tilde{\boldsymbol{\Gamma}}_{\bar{z}_{1} \bar{z}_{2}}^{H}
$$

where

$$
\begin{aligned}
\tilde{\boldsymbol{\Gamma}}_{\bar{z}_{1}} & =\left(\begin{array}{cc}
\boldsymbol{\Lambda}_{r} & \overline{\boldsymbol{\Omega}}_{11} \\
\overline{\boldsymbol{\Omega}}_{11}^{*} & \boldsymbol{\Lambda}_{r}
\end{array}\right), \\
\tilde{\boldsymbol{\Gamma}}_{\bar{z}_{2}} & =\left(\begin{array}{cc}
\boldsymbol{\Lambda}_{r} & \overline{\boldsymbol{\Omega}}_{22} \\
\overline{\boldsymbol{\Omega}}_{22}^{*} & \boldsymbol{\Lambda}_{r}
\end{array}\right), \\
\tilde{\boldsymbol{\Gamma}}_{\bar{z}_{1} \bar{z}_{2}} & =\left(\begin{array}{ll}
\overline{\boldsymbol{\Sigma}}_{12} & \overline{\boldsymbol{\Omega}}_{12} \\
\overline{\boldsymbol{\Omega}}_{21}^{H} & \overline{\boldsymbol{\Sigma}}_{21}^{T}
\end{array}\right),
\end{aligned}
$$


and $\overline{\boldsymbol{\Sigma}}_{12}=\mathbf{U}_{r, 1}^{H} \boldsymbol{\Sigma}_{12} \mathbf{U}_{r, 2}=\overline{\boldsymbol{\Sigma}}_{21}^{H}, \overline{\boldsymbol{\Omega}}_{12}=\mathbf{U}_{r, 1}^{H} \boldsymbol{\Omega}_{12} \mathbf{U}_{r, 2}^{*}=$ $\overline{\boldsymbol{\Omega}}_{21}^{T}$ and $\tilde{\mathbf{z}}_{2} \stackrel{\text { def }}{=}\left(\overline{\mathbf{z}}_{2}^{H} \overline{\mathbf{z}}_{2}^{T}\right)^{H}, \tilde{\boldsymbol{\mu}}_{k} \stackrel{\text { def }}{=}\left(\overline{\boldsymbol{\mu}}_{k}^{H} \overline{\boldsymbol{\mu}}_{k}^{T}\right)^{H}, \mathrm{k}=1,2$. Exploiting the block structure of the matrices given by (68), the parameter vector $\boldsymbol{\mu}_{\bar{z}_{1} \mid \bar{z}_{2}}$ is a $\left(r_{1} \times 1\right)$ vector of the conditional augmented $\left(2 r_{1} \times 1\right)$ vector $(65)$ and using the matrix inversion lemma, and after some algebraic manipulations,

$$
\boldsymbol{\mu}_{\bar{z}_{1} \mid \bar{z}_{2}}=\overline{\boldsymbol{\mu}}_{1}+\overline{\mathbf{E}}\left(\overline{\mathbf{z}}_{2}-\overline{\boldsymbol{\mu}}_{2}\right)+\overline{\mathbf{F}}\left(\overline{\mathbf{z}}_{2}^{*}-\overline{\boldsymbol{\mu}}_{2}^{*}\right),
$$

where

$$
\begin{aligned}
\overline{\mathbf{E}} & =\left(\overline{\boldsymbol{\Sigma}}_{12}-\overline{\boldsymbol{\Omega}}_{12} \boldsymbol{\Lambda}_{r}^{-1} \overline{\boldsymbol{\Omega}}_{22}^{H}\right) \mathbf{P}_{\bar{z}_{2}}^{-*} \\
\overline{\mathbf{F}} & =\left(\overline{\boldsymbol{\Omega}}_{12}-\overline{\boldsymbol{\Sigma}}_{12} \boldsymbol{\Lambda}_{r}^{-1} \overline{\boldsymbol{\Omega}}_{22}\right) \mathbf{P}_{\bar{z}_{2}}^{-1}
\end{aligned}
$$

and $\mathbf{P}_{\bar{z}_{2}} \stackrel{\text { def }}{=} \boldsymbol{\Lambda}_{r_{2}}-\overline{\boldsymbol{\Omega}}_{22}^{H} \boldsymbol{\Lambda}_{r_{2}}^{-1} \overline{\boldsymbol{\Omega}}_{22}$ is the Schur complement of $\tilde{\boldsymbol{\Gamma}}_{\bar{z}_{2}}$.

Similarly, the matrices $\overline{\boldsymbol{\Sigma}}_{11.2}$ and $\overline{\boldsymbol{\Omega}}_{11.2}$ are respectively the top left $\left(r_{1} \times r_{1}\right)$ submatrix and top right $\left(r_{1} \times r_{1}\right)$ submatrix of the conditional augmented scattered $\left(2 r_{1} \times 2 r_{1}\right)$ matrix (66). Using the matrix inversion lemma, and after some algebraic manipulations,

$$
\begin{aligned}
& \overline{\boldsymbol{\Sigma}}_{11.2}=\boldsymbol{\Lambda}_{r_{1}}-\overline{\mathbf{E}} \overline{\boldsymbol{\Sigma}}_{12}^{H}-\overline{\mathbf{F}} \overline{\boldsymbol{\Omega}}_{12}^{H} \\
& \overline{\boldsymbol{\Omega}}_{11.2}=\overline{\boldsymbol{\Omega}}_{11}-\overline{\mathbf{E}} \overline{\boldsymbol{\Omega}}_{21}-\overline{\mathbf{F}} \overline{\boldsymbol{\Sigma}}_{12}^{T} .
\end{aligned}
$$

Using the fact that $\boldsymbol{\Sigma}_{22}^{\#}=\mathbf{U}_{r, 2} \boldsymbol{\Lambda}_{r_{2}}^{-1} \mathbf{U}_{r, 2}^{H}, \mathbf{P}_{\bar{z}_{2}}$ can be expressed as

$$
\begin{aligned}
\mathbf{P}_{\bar{z}_{2}} & =\boldsymbol{\Lambda}_{r_{2}}-\overline{\mathbf{\Omega}}_{22}^{H} \boldsymbol{\Lambda}_{r_{2}}^{-1} \overline{\mathbf{\Omega}}_{22} \\
& =\mathbf{U}_{r, 2}^{T}\left(\mathbf{U}_{r, 2}^{*} \boldsymbol{\Lambda}_{r, 2} \mathbf{U}_{r, 2}^{T}-\mathbf{\Omega}_{22}^{H} \mathbf{U}_{r, 2} \boldsymbol{\Lambda}_{r_{2}}^{-1} \mathbf{U}_{r, 2}^{H} \boldsymbol{\Omega}_{22}\right) \mathbf{U}_{r, 2}^{*} \\
& =\mathbf{U}_{r, 2}^{T} \mathbf{P}_{z_{2}} \mathbf{U}_{r, 2}^{*}
\end{aligned}
$$

with $\mathbf{P}_{z_{2}} \stackrel{\text { def }}{=} \boldsymbol{\Sigma}_{22}^{*}-\boldsymbol{\Omega}_{22}^{H} \boldsymbol{\Sigma}_{22}^{\#} \boldsymbol{\Omega}_{22}$. In a similar way, we obtain

$$
\begin{aligned}
\overline{\mathbf{E}} & =\mathbf{U}_{r, 1}^{H}\left(\boldsymbol{\Sigma}_{12}-\boldsymbol{\Omega}_{12} \boldsymbol{\Sigma}_{22}^{* \#} \overline{\boldsymbol{\Omega}}_{22}^{H}\right) \mathbf{U}_{r, 2}\left(\mathbf{U}_{r, 2}^{H} \mathbf{P}_{z_{2}}^{*} \mathbf{U}_{r, 2}\right)^{-(73)} \\
\overline{\mathbf{F}} & =\mathbf{U}_{r, 1}^{H}\left(\boldsymbol{\Omega}_{12}-\boldsymbol{\Sigma}_{12} \boldsymbol{\Sigma}_{22}^{\#} \overline{\boldsymbol{\Omega}}_{22}\right) \mathbf{U}_{r, 2}^{*}\left(\mathbf{U}_{r, 2}^{T} \mathbf{P}_{z_{2}} \mathbf{U}_{r, 2}^{*}\right)^{-\nmid}(74)
\end{aligned}
$$

Using (73)-(74) and $\mathbf{P}_{z_{2}}^{\#}=\mathbf{U}_{r, 2}^{*}\left(\mathbf{U}_{r, 2}^{T} \mathbf{P}_{z_{2}} \mathbf{U}_{r, 2}^{*}\right)^{-1} \mathbf{U}_{r, 2}^{T},(68)$ can be written as

$$
\boldsymbol{\mu}_{\bar{z}_{1} \mid \bar{z}_{2}}=\mathbf{U}_{r, 1}^{H}\left(\boldsymbol{\mu}_{1}+\mathbf{E}\left(\mathbf{z}_{2}-\boldsymbol{\mu}_{2}\right)+\mathbf{F}\left(\mathbf{z}_{2}^{*}-\boldsymbol{\mu}_{2}^{*}\right)\right)
$$

where

$$
\begin{aligned}
& \mathbf{E} \stackrel{\text { def }}{=}\left(\boldsymbol{\Sigma}_{12}-\boldsymbol{\Omega}_{12} \boldsymbol{\Sigma}_{22}^{* \#} \overline{\boldsymbol{\Omega}}_{22}^{H}\right) \mathbf{P}_{z_{2}}^{* \#} \\
& \mathbf{F} \stackrel{\text { def }}{=}\left(\boldsymbol{\Omega}_{12}-\boldsymbol{\Sigma}_{12} \boldsymbol{\Sigma}_{22}^{\#} \overline{\boldsymbol{\Omega}}_{22}\right) \mathbf{P}_{z_{2}}^{\#}
\end{aligned}
$$

We conclude, then, that

$$
\boldsymbol{\mu}_{z_{1} \mid z_{2}}=\boldsymbol{\mu}_{1}+\mathbf{E}\left(\mathbf{z}_{2}-\boldsymbol{\mu}_{2}\right)+\mathbf{F}\left(\mathbf{z}_{2}^{*}-\boldsymbol{\mu}_{2}^{*}\right)
$$

In the same way, using (71), we obtain

$$
\begin{aligned}
& \overline{\boldsymbol{\Sigma}}_{11.2}=\mathbf{U}_{r, 1}^{H}\left(\boldsymbol{\Sigma}_{11}-\mathbf{E} \boldsymbol{\Sigma}_{12}^{H}-\mathbf{F} \boldsymbol{\Omega}_{12}^{H}\right) \mathbf{U}_{r, 1} \\
& \overline{\boldsymbol{\Omega}}_{11.2}=\mathbf{U}_{r, 1}^{H}\left(\boldsymbol{\Omega}_{11}-\mathbf{E} \boldsymbol{\Omega}_{12}^{T}-\mathbf{F} \boldsymbol{\Sigma}_{12}^{T}\right) \mathbf{U}_{r, 1}^{*} .
\end{aligned}
$$

We conclude that

$$
\begin{aligned}
& \boldsymbol{\Sigma}_{11.2}=\boldsymbol{\Sigma}_{11}-\mathbf{E} \boldsymbol{\Sigma}_{12}^{H}-\mathbf{F} \boldsymbol{\Omega}_{12}^{H} \\
& \boldsymbol{\Omega}_{11.2}=\boldsymbol{\Omega}_{11}-\mathbf{E} \boldsymbol{\Omega}_{12}^{T}-\mathbf{F} \boldsymbol{\Sigma}_{12}^{T} .
\end{aligned}
$$

\section{E Proof of result 9}

Since $\mathbf{z}$ possesses a stochastic representation (28), it follows that the quadratic form in (32) can be simplified as

$$
\tilde{\mathbf{z}}_{0}^{H} \tilde{\mathbf{Q}} \tilde{\mathbf{z}}_{0}=\tau \tilde{\mathbf{n}}^{H} \tilde{\mathbf{Q}} \tilde{\mathbf{n}} .
$$

Because the $m$-variate NC-CN r.v. $\mathbf{n}$ is a $2 m$-variate real Gaussian r.v. $\overline{\mathbf{n}} \stackrel{\text { def }}{=}\left(\begin{array}{cc}\mathbf{z}_{r}^{T} & \mathbf{z}_{i}^{T}\end{array}\right)^{T} \in \mathbb{R}^{2 m}$ with $\tilde{\mathbf{n}}=\mathbf{T} \overline{\mathbf{n}}$ and $\underset{\text { comes }}{\mathbf{T}} \stackrel{\text { def }}{=}\left(\begin{array}{cc}\mathbf{I} & j \mathbf{I} \\ \mathbf{I} & -j \mathbf{I}\end{array}\right)$, the quadratic term $\tilde{\mathbf{n}}^{H} \tilde{\mathbf{Q}} \tilde{\mathbf{n}}$ in (80) be-

$$
\tilde{\mathbf{n}}^{H} \tilde{\mathbf{Q}} \tilde{\mathbf{n}}=\overline{\mathbf{n}}^{T} \overline{\mathbf{Q}} \overline{\mathbf{n}},
$$

where $\overline{\mathbf{Q}} \stackrel{\text { def }}{=} \mathbf{T}^{H} \tilde{\mathbf{Q}} \mathbf{T}$. Using the fact that $\overline{\mathbf{n}} \sim \mathcal{N}_{2 m}\left(\mathbf{0}, \overline{\boldsymbol{\Gamma}}_{r}\right)$ where $\overline{\boldsymbol{\Gamma}}_{r}=\frac{1}{4} \mathbf{T}^{H} \tilde{\boldsymbol{\Gamma}} \mathbf{T}$, (81) can also be written as

$$
\overline{\mathbf{n}}^{T} \overline{\mathbf{Q}} \overline{\mathbf{n}}=\overline{\mathbf{n}}_{\gamma}^{T} \overline{\mathbf{Q}}_{\gamma} \overline{\mathbf{n}}_{\gamma},
$$

with $\overline{\mathbf{n}}_{\gamma} \stackrel{\text { def }}{=} \overline{\boldsymbol{\Gamma}}_{r}^{-1 / 2} \overline{\mathbf{n}} \sim \mathcal{N}_{2 m}(\mathbf{0}, \mathbf{I})$ and $\overline{\mathbf{Q}}_{\gamma} \stackrel{\text { def }}{=} \overline{\boldsymbol{\Gamma}}_{r}^{1 / 2} \overline{\mathbf{Q}} \overline{\boldsymbol{\Gamma}}_{r}^{1 / 2}$. The singular eigenvalue decomposition of $\mathbf{Q}_{\gamma}$ can be written as $\overline{\mathbf{Q}}_{\gamma}=\mathbf{U}_{\gamma} \boldsymbol{\Lambda}_{\gamma} \mathbf{U}_{\gamma}^{T}$ where $\boldsymbol{\Lambda}_{\gamma} \stackrel{\text { def }}{=} \operatorname{Diag}\left(\lambda_{1}, \ldots, \lambda_{q}\right)$ is a diagonal matrix containing only the $q$ nonzero real eigenvalues $\lambda_{1} \geq \lambda_{1} \geq \ldots \geq \lambda_{q}$ and the columns of $\mathbf{U}_{\gamma}$ are the associated orthonormal eigenvectors. Hence, from (82) and (81), the $\tilde{\mathbf{z}}_{0}^{H} \tilde{\mathbf{Q}} \tilde{\mathbf{z}}_{0}$ given by (80) becomes

$$
\tilde{\mathbf{z}}_{0}^{H} \tilde{\mathbf{Q}} \tilde{\mathbf{z}}_{0}=\tau \sum_{l=1}^{q} \lambda_{l}\left|\check{n}_{l}\right|^{2},
$$

where $\check{\mathbf{n}}=\left(\check{n}_{1}, \ldots, \check{n}_{q}\right)^{T} \stackrel{\text { def }}{=} \mathbf{U}_{\gamma}^{T} \overline{\mathbf{n}}_{\gamma} \sim \mathcal{N}_{q}(\mathbf{0}, \mathbf{I})$. Since $\left|\check{n_{l}}\right|^{2} \sim \chi_{1}^{2}$, the quadratic form in (83) is a weighted sum of independent central Chi-squares r.v.'s with one degree of freedom multiplied by a scaled texture r.v. Therefore, result 9 is proved.

\section{REFERENCES}

[1] E. Ollila and V. Koivunen, "Robust antenna array processing using M-estimators of pseudo-covariance," in Proc. 14th IEEE Int. Symp. Personal, Indoor, Mobile Radio Commun. (PIMRC), Beijing, China, Sep. 710, pp. 2659-663, 2003.

[2] M. Mahot, F. Pascal, P. Forster, and J. P. Ovalez, "Asymptotic Properties of Robust Complex Covariance Matrix Estimates," IEEE Trans. Signal Process., vol. 61, no. 13, pp. 3348-3356, July 2013.

[3] Y. Abramovich and O. Besson, "Regularized Covariance Matrix Estimation in Complex Elliptically Symmetric Distributions Using the Expected Likelihood Approach-Part 1: The OverSampled Case," IEEE Trans. Signal Process., vol. 61, no. 23, pp. 5807-5818, Dec. 2013.

[4] E. Ollila and D. E. Tyler, "Regularized M-estimators of scatter matrix," IEEE Trans. Signal Process., vol. 62, no. 22, pp. 60596070, Nov. 2014. 
[5] E. Ollila, J. Eriksson, and V. Koivunen, "Complex elliptically symmetric random variables generation, characterization, and circularity tests," IEEE Trans. Signal Process., vol. 59, no. 1, pp. 58-69, Jan. 2011.

[6] M. S. Greco, S. Fortunati and F. Gini, "Maximum likelihood covariance matrix estimation for complex elliptically symmetric distributions under mismatched conditions," ELSEVIER Signal Process., vol. 104, no. 0, pp. 381-386, Nov. 2014.

[7] F. Pascal and A. Renaux, "Statistical analysis of the covariance matrix MLE in K-distributed clutter," Elsevier Signal Processing, vol. 90, pp. 1165-1175, Apr. 2010.

[8] X. Zhang, M. N. El Korso and M. Pesavento, "MIMO radar target localization and performance evaluation under SIRP clutter”, Signal Process. Journal, Elsevier, vol. 130, Pp. 217-232, Jan. 2017

[9] P. R. Krishnaiah and J. Lin, "Complex elliptically symmetric distributions," Comm. Statist. - Th. and Meth., vol. 15, pp. 3693$3718,1986$.

[10] E. Ollila, D. Tyler, V. Koivunen, and H. Poor, "Complex elliptically symmetric distributions: Survey, new results and applications," IEEE Trans. Signal Process., vol. 60, no. 11, pp. 5597-5625, Nov. 2012.

[11] E. Ollila, V. Koivunen, "Generalized complex elliptical distributions," in Proc. SAM Workshop, pp. 460-464, July 2004.

[12] N. R. Goodman, "Statistical analysis based on certain multivariate complex Gaussian distribution (an introduction)," $A n$ nals Math. Statist., vol. 34, pp. 152-177, 1963.

[13] R. A.Wooding, "The multivariate distribution of complex normal variables," Biometrika, vol. 43, pp. 212-215, 1956.

[14] B. Picinbono, "Second order complex random vectors and normal distributions," IEEE Trans. Signal Process., vol. 44, no. 10, pp. 2637-2640, 1996.

[15] M. Novey, T. Adali, and A. Roy, "Circularity and Gaussianity detection using the complex generalized Gaussian distribution," IEEE Signal Process. Lett., vol. 16, no. 11, pp. 993-996, 2009.

[16] P. Charg, Y. Wang, and J. Saillard, "A non-circular sources direction finding methods using polynomial rooting," Signal Process., vol. 81, pp. 1765-1770, 2001.

[17] M. Haardt and F. Romer, "Enhancements of unitary ESPRIT for noncircular sources," in Proc. Int. Conf. Acoust., Speech Signal Process. (ICASSP04), Montreal, Canada, May 2004.

[18] H. Abeida and J.-P. Delmas "MUSIC-like estimation of direction of arrival for noncircular sources," IEEE Trans. Signal Process., vol. 54, no. 7, pp.2678-2690, 2006.

[19] J. Eriksson and V. Koivunen, "Complex random vectors and ICA models: Identifiability, uniqueness and seperability," IEEE Trans. Inf. Theory, vol. 52, no. 3, pp. 1017-1029, 2006.

[20] M. Novey and T. Adal, "On extending the complex fastICA algorithm to noncircular sources," IEEE Trans. Signal Process., vol. 56, no. 5, pp. 2148-2154, Apr. 2008.

[21] E. Ollila and V. Koivunen, "Complex ICA using generalized uncorrelating transform," Signal Process., vol. 89, no. 5, pp. 365-377, 2009.
[22] A. van den Bos, "The multivariate complex normal distributionA generalization," IEEE Trans. Inf. Theory, vol. 41, no. 2, pp. 537-539, 1995.

[23] E. Conte, M. Lops, and G. Ricci, “Asymptotically optimum radar detection in compound-Gaussian clutter," IEEE Trans. Aerosp. Electron. Syst., vol. 31, no. 2, pp. 617-624, 1995.

[24] F. Gini, "Sub-optimum coherent radar detection in a mixture of -distributed and Gaussian clutter," Proc. Inst. Electr. Eng.Radar, Sonar Navig., vol. 144, no. 1, pp. 39-48, 1997.

[25] M. Novey, T. Adali, and A. Roy, "A complex generalized Gaussian distribution-Characterization, generation, and estimation," IEEE Trans. Signal Process., vol. 58, no. 3, pp. 1427-1433, 2010.

[26] J. A. Díaz-García, V. Leiva-Sánchez and M. Galea, "Singular elliptic distribution: density and applications," Commun. Statist., vol. 31 , no. 5, pp. 665-681, 2002.

[27] B. Picinbono and P. Bondon, "Second-order statistics of complex signals," IEEE Trans. on Sig. Process., vol. 45, no. 2, pp. 411-420, 1997.

[28] B. Picinbono and P. Chevalier, "Widely linear estimation with complex Data," IEEE Trans. on Sig. Proces., vol. 43, no. 8, pp. 2030-2033, 1995.

[29] P. J. Schreier, L. L. Scharf, and C. T. Mullis, "Detection and estimation of improper complex random signals," IEEE Trans. on Inform. on Theory, vol. 51, no .1, pp. 306-312, 2005.

[30] P. J. Schreier and L. L. Scharf, Statistical signal processing of complex-valued data: The theory of improper and noncircular signals, 2010, Cambridge Univ. Press.

[31] T. Adali, P. Schreier, and L. Scharf, "Complex-valued signal processing: The proper way to deal with impropriety," IEEE Trans. on Signal Proc., Vol. 59, No. 11, pp. 5101-5125, Nov 2011.

[32] O.Lang and M. Huemer, "Classical widely linear estimation of real valued parameter vectors in complex valued environments," arXiv:1704.08825 [math.ST]

[33] S. Kanna, S. Talebi, and D. Mandic, "Diffusion widely linear adaptive estimation of system frequency in distributed power grids," In Proceedings of the IEEE International Energy Conference, Leuven, Belgium, 8 April 2014, pp. 772-778.

[34] D. Mandic and V. S. L. Goh, Complex valued nonlinear adaptive filters: Non circularity, widely linear and neural models. Wiley, 2009

[35] R. A. Horn and C. A. Johnson, Matrix Analysis, Cambridge University Press, New York, 1985.

[36] P. J. Schreier, L. L. Scharf, and A. Hanssen, "A generalized likelihood ratio test for impropriety of complex signals," IEEE Signal Processing Letters, vol. 13, no. 7, pp. 433-436, 2006.

[37] J. Eriksson, E. Ollila, and V. Koivunen, "Essential statistics and tools for complex random variables," IEEE Trans. Signal Processing, vol. 58, no. 10, pp. 5400-5408, 2010.

[38] J. P. Delmas and H. Abeida, "Stochastic Cramér-Rao bound for noncircular signals with application to DOA estimation," IEEE Trans. Signal Process., vol. 52, no. 11, pp. 3192-3199, 2004. 
[39] B. Loesch and B. Yang, "Cramér-rao bound for circular and noncircular complex independent component analysis," IEEE Trans. Signal Process., vol. 61, no. 2, pp. 365-379, Jan. 2013.
[40] J.R. Schott, Matrix Analysis for Statistics, Wiley, New York, 1980. 